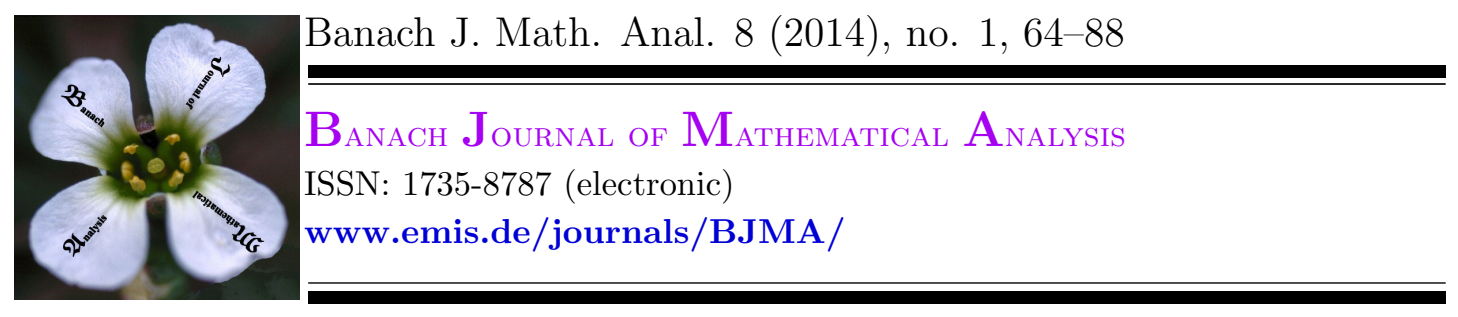

\title{
COMPOSITION OPERATORS BETWEEN WEIGHTED BERGMAN SPACES WITH ADMISSIBLE BÉKOLLÉ WEIGHTS
}

\author{
AJAY K. SHARMA ${ }^{1}$ AND SEI-ICHIRO UEKI ${ }^{2 *}$ \\ Communicated by T. Sugawa
}

\begin{abstract}
We study composition operators acting between weighted Bergman spaces with admissible Békollé weights. The boundedness and compactness of composition operators are characterized in terms of the generalized Nevanlinna counting function associated with the inducing map of the composition operator and the associated weight function of Bergman space. For a special case, we also give the estimate of the essential norm.
\end{abstract}

\section{INTRODUCTION}

Let $\mathbb{D}$ denote the open unit disk in the complex plane and $H(\mathbb{D})$ the space of all analytic functions on $\mathbb{D}$. For a given non-negative integrable function $\sigma$ on $\mathbb{D}$, we denote $L^{p}(\sigma d A)(p>0)$ the space of measurable functions $f$ with

$$
\|f\|_{\sigma}^{p}=\int_{\mathbb{D}}|f(z)|^{p} \sigma(z) d A(z)<\infty,
$$

where $d A$ is the normalized Lebesgue measure on $\mathbb{D}$, and $A^{p}(\sigma d A)=L^{p}(\sigma d A) \cap$ $H(\mathbb{D})$. If $\sigma(z)=\left(1-|z|^{2}\right)^{\alpha}(\alpha>-1)$, then $A^{p}(\sigma d A)$ is the well-known weighted Bergman space $A_{\alpha}^{p}$. In this paper, we will consider the space $A^{p}(\sigma d A)$ and study the composition operator acting on this space.

For any analytic self-map $\phi: \mathbb{D} \rightarrow \mathbb{D}$, the composition operator $C_{\phi}: H(\mathbb{D}) \rightarrow$ $H(\mathbb{D})$ is defined by $C_{\phi} f=f \circ \phi$. One of interesting subjects on studies of this

Date: Received: 9 January 2013; Accepted: 25 April 2013.

* Corresponding author.

2010 Mathematics Subject Classification. Primary 47B33; Secondary 30H20.

Key words and phrases. Composition operators, Bergman spaces, generalized Nevanlinna counting functions. 
operator is to characterize its operator theoretic property in terms of the function theoretic property of $\phi$. For the Hardy space $H^{p}$ or the weighted Bergman space $A_{\alpha}^{p}$, a consequence of the Littlewood subordination principle verifies that all composition operators are bounded on $H^{p}$ or $A_{\alpha}^{p}$. However, it is known that every analytic self-map of $\mathbb{D}$ does not induce a compact composition operator on $H^{p}$ or $A_{\alpha}^{p}$. The classical Nevanlinna counting function $N_{\phi}$ plays a key role in the study on compact composition operator on $H^{2}$. For a given analytic self-map $\phi$ of $\mathbb{D}, N_{\phi}$ is defined by

$$
N_{\phi}(z)=\sum_{w \in \phi^{-1}(z)} \log \frac{1}{|w|} \quad(z \in \mathbb{D} \backslash\{\phi(0)\}),
$$

where we understand that $N_{\phi}(z)=0$ for $z \notin \phi(\mathbb{D})$ and $w \in \phi^{-1}(z)$ is repeated according to the multiplicity of zeros of $\phi-z$. This $N_{\phi}$ was used for establishing a formula for the essential norm of $C_{\phi}$ on $H^{2}$ by Shapiro [11]. As a consequence, he proved that the compactness of $C_{\phi}$ on $H^{2}$ is characterized by the condition $N_{\phi}(z)=o(-\log |z|)$ as $|z| \rightarrow 1$. Furthermore, he generalized $N_{\phi}$ as follows

$$
N_{\phi, \gamma}(z)=\sum_{w \in \phi^{-1}(z)}\left\{\log \frac{1}{|w|}\right\}^{\gamma} \quad(\gamma>0, z \in \mathbb{D} \backslash\{\phi(0)\})
$$

and also characterized the compactness of $C_{\phi}$ on $A_{\alpha}^{2}$.

Smith [12] used the above generalized counting function to study composition operators $C_{\phi}$ acting between different weighted Bergman spaces $A_{\alpha}^{p}$. When $0<$ $p \leq q<\infty$, Smith proved that $C_{\phi}: A_{\alpha}^{p} \rightarrow A_{\beta}^{q}$ is bounded (or compact) if and only if $N_{\phi, \beta+2}(z)=O\left((-\log |z|)^{(\alpha+2) q / p}\right)\left(\right.$ or $\left.o\left((-\log |z|)^{(\alpha+2) q / p}\right)\right)$ as $|z| \rightarrow 1$. Since this result include the cases $A_{-1}^{p}=H^{p}$ or $A_{-1}^{q}=H^{q}$, he also characterized the boundedness and compactness of $C_{\phi}$ acting between the Hardy space and the Bergman space. Pérez-González, Rättyä and Vukotić [7] also considered the same characterization problem for the compactness of $C_{\phi}: A_{\alpha}^{p} \rightarrow A_{\beta}^{q}(0<$ $p \leq q<\infty)$. They proved that the condition $d \mu(z)=N_{\phi, \beta+2}(z) d A(z)$ is a vanishing $2+q(\alpha+2) / p$-Carleson measure also characterizes the compactness of $C_{\phi}: A_{\alpha}^{p} \rightarrow A_{\beta}^{q}$.

On the other hand, Smith and Yang [13] considered the case $C_{\phi}: A_{\alpha}^{p} \rightarrow A_{\beta}^{q}$ when $0<q<p$. The boundedness of this case has a relation to the Carleson-type embedding theorem. By using a method which is based on Khinchine's inequality and the atomic decomposition of $f \in A_{\alpha}^{p}$, they proved that $C_{\phi}: A_{\alpha}^{p} \rightarrow A_{\beta}^{q}(q<p)$ is bounded if and only if the function

$$
\mathbb{D} \ni z \mapsto \frac{N_{\phi, \beta+2}(z)}{\left(1-|z|^{2}\right)^{2+\alpha}}
$$

belongs to the space $L^{\frac{p}{p-q}}\left(d A_{\alpha}\right)$. They also showed that every bounded composition operator $C_{\phi}: A_{\alpha}^{p} \rightarrow A_{\beta}^{q}(q<p)$ is also compact.

These results suggest a problem that what conditions on $\phi$ characterize the boundedness and compactness of $C_{\phi}$ acting between different weighted Bergman spaces $A^{p}(\sigma d A)$ with more general weight functions $\sigma$. Recently, Constantin [4] studied this problem. Constantin's characterizations are based on Carleson-type 
measure conditions for the pull-back measure induced by $\phi$ (see Theorem 2.11 below). Our aim in the present paper is to give another type characterization for the boundedness and compactness of $C_{\phi}$ acting between different spaces $A^{p}\left(\sigma_{1} d A\right)$ and $A^{q}\left(\sigma_{2} d A\right)$. In order to investigate this problem, we shall need the following counting function.

Definition. Let $\phi$ be an analytic self-map of $\mathbb{D}$ and $\sigma$ a weight function on $\mathbb{D}$. We define the function $\mathcal{N}_{\phi, \sigma}$ as follows.

$$
\mathcal{N}_{\phi, \sigma}(z)=\sum_{w \in \phi^{-1}(z)} \sigma(w) \quad(z \in \mathbb{D} \backslash\{\phi(0)\}) .
$$

As in the classical Nevanlinna counting function $N_{\phi}$, we understand that $\mathcal{N}_{\phi, \sigma}(z)=$ 0 for $z \notin \phi(\mathbb{D})$ and $w \in \phi^{-1}(z)$ is repeated according to the multiplicity of zeros of $\phi-z$. Conventionally, we consider that $\mathcal{N}_{\phi, \sigma}(z)=0$ if $z=\phi(0)$. When $\sigma(z)=-\log |z|$, this $\mathcal{N}_{\phi, \sigma}$ coincides with $N_{\phi}$. So we call $\mathcal{N}_{\phi, \sigma}$ a generalized Nevanlinna counting function associated to $\phi$ and $\sigma$.

This generalized Nevanlinna counting function $\mathcal{N}_{\phi, \sigma}$ was first introduced by Kellay and Lefèvre in [8]. They used $\mathcal{N}_{\phi, \sigma}$ to study the compactness of $C_{\phi}$ on the weighted Dirichlet-type space $\mathcal{H}_{\sigma}$ which consists of all analytic functions $f$ on $\mathbb{D}$ such that $\int_{\mathbb{D}}\left|f^{\prime}(z)\right|^{2} \sigma(z) d A(z)<\infty$. They gave the characterization for the compactness of $C_{\phi}$ on the space $\mathcal{H}_{\sigma}$ by the growth condition of the generalized Nevanlinna counting function $\mathcal{N}_{\phi, \sigma}$. Their results inspired us to study the composition operator on $A^{p}(\sigma d A)$ and gave suggestions for the method of characterizations for the boundedness and compactness of $C_{\phi}: A^{p}\left(\sigma_{1} d A\right) \rightarrow A^{p}\left(\sigma_{2} d A\right)$.

The main result of the paper is to characterize the bounded and compact composition operator from $A^{p}\left(\sigma_{1} d A\right)$ into $A^{q}\left(\sigma_{2} d A\right)$ in terms of the behavior of the above generalized Nevanlinna counting function. In section 3 , we will consider the operator $C_{\phi}: A^{p}\left(\sigma_{1} d A\right) \rightarrow A^{q}\left(\sigma_{2} d A\right)$ when $0<p \leq q<\infty$. The first result in this section says that the boundedness is characterized by the growth condition of this counting function. The second one is to estimate the essential norm of $C_{\phi}$ under some restricted assumptions on $p$ and $q$. Once this estimate is accomplished, we get as a consequence the characterization of the compactness for $0<p \leq q<\infty$. In section 4, we will investigate the case $0<q<p<\infty$. Applications of well-known Khinchine's inequality and the Hardy-Littlewood maximal function play an important role in our argument of this section. The result shows that the integrability condition of the generalized counting function characterizes the boundedness of $C_{\phi}$. Furthermore we prove that the bounded composition operator $C_{\phi}: A^{p}\left(\sigma_{1} d A\right) \rightarrow A^{q}\left(\sigma_{2} d A\right)$ is also compact when $0<q<p<\infty$.

Throughout this paper, the notation $a \lesssim b$ means that there exists a positive constant $C$ such that $a \leq C b$. Moreover, if both $a \lesssim b$ and $a \gtrsim b$ hold, then one says that $a \approx b$.

\section{Preliminaries}

In this section, we introduce an admissible Békollé weight function $\sigma$ and a composition operator on weighted Bergman spaces $A^{p}(\sigma d A)$. We shall need some lemmas on the space $A^{p}(\sigma d A)$ or the weight $\sigma$, so we also describe them. 
For each $a \in \mathbb{D}$, let $\varphi_{a}(z)$ be the Möbius transformation of $\mathbb{D}$ interchanging $a$ and 0 , that is $\varphi_{a}(z)=(a-z) /(1-\bar{a} z)$. Recall that the pseudohyperbolic metric $\rho$ on $\mathbb{D}$ is defined by $\rho(z, a)=\left|\varphi_{a}(z)\right|$, and the pseudohyperbolic disk $E(a, r)$ is the set

$$
E(a, r)=\{z \in \mathbb{D}: \rho(z, a)<r\},
$$

for $a \in \mathbb{D}$ and $r \in(0,1)$.

2.1. Admissible Békollé weight. For each $\alpha>-1$, let $d A_{\alpha}$ denote the normalized measure on $\mathbb{D}$ defined by $d A_{\alpha}(z)=(\alpha+1)\left(1-|z|^{2}\right)^{\alpha} d A(z)$. For $p>1$ and $\alpha>-1$, the class $B_{p}(\alpha)$ consists of all weight functions $\sigma$ with the property that there is a constant $C>0$ such that for every $S(a)=\left\{\varphi_{a}(z): \operatorname{Re}(z \bar{a}) \leq 0\right\}, a \in \mathbb{D}$,

$$
\left(\int_{S(a)} \sigma d A_{\alpha}\right) \cdot\left(\int_{S(a)} \sigma^{-\frac{p^{\prime}}{p}} d A_{\alpha}\right)^{\frac{p}{p^{\prime}}} \leq C\left\{A_{\alpha}(S(a))\right\}^{p},
$$

where $p^{\prime}$ is the conjugate exponent of $p$. Note that we put $S(0)=\mathbb{D}$.

Békollé [2] proved that this condition characterizes the boundedness of the Bergman projection $P_{\alpha}$ defined by

$$
P_{\alpha} f(z)=\int_{\mathbb{D}} \frac{f(w)}{(1-\bar{w} z)^{\alpha+2}} d A_{\alpha}(w)
$$

on $L^{p}(\sigma d A)$.

Theorem 2.1 ([2] Békollé). Let $1<p<\infty$ and $\alpha>-1$. For a weight function $\sigma$, the following conditions are equivalent:

(i) $P_{\alpha}$ is a bounded projection from $L^{p}(\sigma d A)$ onto $A^{p}(\sigma d A)$.

(ii) The sublinear operator $\widetilde{P_{\alpha}}$ defined by

$$
\widetilde{P_{\alpha}} f(z)=\int_{\mathbb{D}} \frac{|f(w)|}{|1-\bar{w} z|^{\alpha+2}} d A_{\alpha}(w)
$$

is bounded on $L^{p}(\sigma d A)$.

(iii) The z-variable function $\sigma(z) /\left(1-|z|^{2}\right)^{\alpha}$ belongs to $B_{p}(\alpha)$.

This result is very useful to study on the space $A^{p}(\sigma d A)$. For instance, Luecking [9] showed the following dual relation of $A^{p}(\sigma d A)$.

Theorem 2.2 ([9] Luecking). Let $1<p<\infty$ and $\alpha>-1$. If a weight function $\sigma$ satisfies the condition $\sigma(z) /\left(1-|z|^{2}\right)^{\alpha} \in B_{p}(\alpha)$, then the dual space of $A^{p}(\sigma d A)$ can be identified with $A^{p^{\prime}}\left(\sigma^{-\frac{p^{\prime}}{p}} d A_{\alpha p^{\prime}}\right)$ under the integral pairing

$$
\langle f, g\rangle_{\alpha}=\int_{\mathbb{D}} f(z) \overline{g(z)} d A_{\alpha}(z),
$$

where $p^{\prime}$ is the conjugate exponent of $p$.

As an application of Theorem 2.2, we have the following lemma.

Lemma 2.3. Suppose that $1<p<\infty, \alpha>-1$ and a weight function $\sigma$ satisfies $\sigma(z) /\left(1-|z|^{2}\right)^{\alpha} \in B_{p}(\alpha)$. If a bounded sequence $\left\{f_{j}\right\}$ in $A^{p}(\sigma d A)$ converges to 0 uniformly on compact subsets of $\mathbb{D}$, then $\left\{f_{j}\right\}$ also converges to 0 weakly in $A^{p}(\sigma d A)$. 
Proof. Take a function $h \in A^{p^{\prime}}\left(\sigma^{-\frac{p^{\prime}}{p}} d A_{\alpha p^{\prime}}\right)$ where $p^{\prime}$ is the conjugate exponent of $p$. For any $\epsilon>0$, there is a continuous function $g$ with compact support such that

$$
\left[\int_{\mathbb{D}}|\overline{h(z)}-g(z)|^{p^{\prime}} \sigma(z)^{-\frac{p^{\prime}}{p}}\left(1-|z|^{2}\right)^{\alpha p^{\prime}} d A(z)\right]^{\frac{1}{p^{\prime}}}<\epsilon .
$$

By the integral pairing (2.1) in Theorem 2.2, we have that

$$
\begin{aligned}
\left|\left\langle f_{j}, h\right\rangle_{\alpha}\right| & =\left|\int_{\mathbb{D}}\left\{f_{j}(z) \overline{h(z)}-f_{j}(z) g(z)+f_{j}(z) g(z)\right\} d A_{\alpha}(z)\right| \\
& \leq \int_{\mathbb{D}}\left|f_{j}(z)\right||\overline{h(z)}-g(z)| d A_{\alpha}(z)+\int_{\mathbb{D}}\left|f_{j}(z)\right||g(z)| d A_{\alpha}(z) \\
& =(I)+(I I) .
\end{aligned}
$$

By applying the Hölder's inequality to $(I),(2.2)$ gives that

$$
\begin{aligned}
(I) & \leq\left[\int_{\mathbb{D}}\left|f_{j}\right|^{p} \sigma d A\right]^{\frac{1}{p}} \cdot\left[\int_{\mathbb{D}}|\overline{h(z)}-g(z)|^{p^{\prime}} \sigma(z)^{-\frac{p^{\prime}}{p}}\left(1-|z|^{2}\right)^{\alpha p^{\prime}} d A(z)\right]^{\frac{1}{p^{\prime}}} \\
& \leq \epsilon \cdot\left\|f_{j}\right\|_{\sigma}
\end{aligned}
$$

for any $j \geq 1$.

On the other hand, since $g$ has a compact support $\operatorname{supp}(g)$ and $f_{j} \rightarrow 0$ uniformly on $\operatorname{supp}(g)$ as $j \rightarrow \infty$, we see that

$$
(I I)=\int_{\operatorname{supp}(g)}\left|f_{j}(z) \| g(z)\right| d A_{\alpha}(z) \rightarrow 0 \quad(j \rightarrow \infty) .
$$

Thus (2.3), (2.4) and (2.5) show that

$$
\limsup _{j \rightarrow \infty}\left|\left\langle f_{j}, h\right\rangle_{\alpha}\right| \leq \sup _{j \geq 1}\left\|f_{j}\right\|_{\sigma} \epsilon,
$$

for each $h \in A^{p^{\prime}}\left(\sigma^{-\frac{p^{\prime}}{p}} d A_{\alpha p^{\prime}}\right)$. Since $\left\{f_{j}\right\}$ is bounded and $\epsilon>0$ is arbitrarily, this implies that $\left\{f_{j}\right\}$ converges to 0 weakly in $A^{p}(\sigma d A)$.

In this paper, $\sigma$ denotes a non-negative continuous function on $[0,1)$ such that $\sigma(r) \leq 1$ for $r \in[0,1)$. For $z \in \mathbb{D}$ we write $\sigma(z)=\sigma(|z|)$ and call such $\sigma$ a weight function on $\mathbb{D}$. Our arguments in proofs of main results are based on the above results and require some growth conditions on $\sigma$, so we will consider the following conditions.

Definition. A weight function $\sigma$ is called an admissible Békollé weight if $\sigma$ satisfies

$\left(W_{1}\right) \frac{\sigma(z)}{\left(1-|z|^{2}\right)^{\alpha}} \in B_{p_{0}}(\alpha)$ for some $p_{0}>1$ and $\alpha>-1$,

$\left(W_{2}\right) \sigma$ is non-increasing on $[0,1)$,

$\left(W_{3}\right) \sigma(r) /\left(1-r^{2}\right)^{1+\delta}$ is non-decreasing on $[0,1)$ for some $\delta>0$.

Lemma 2.4. Let $p>0$ and $\sigma$ be an admissible Békollé weight function. Then for each $f \in A^{p}(\sigma d A)$, 


$$
\begin{aligned}
|f(z)| \lesssim \frac{\|f\|_{\sigma}}{\sigma(z)^{1 / p}\left(1-|z|^{2}\right)^{2 / p}} \\
\left|f^{\prime}(z)\right| \lesssim \frac{\|f\|_{\sigma}}{\sigma(z)^{1 / p}\left(1-|z|^{2}\right)^{1+2 / p}}
\end{aligned}
$$

Proof. Since $\sigma(z) /\left(1-|z|^{2}\right)^{\alpha} \in B_{p_{0}}(\alpha)$, it follows from Lemma (3.1) in [9] that

$$
|f(z)| \lesssim\left(\int_{E(z, r)} \sigma(w) d A(w)\right)^{-\frac{1}{p}}\|f\|_{\sigma}
$$

for $r \in(0,1)$ and $z \in \mathbb{D}$. For $w \in E(z, r)$, we may assume that $|z| \leq|w|$ without loss of generality. Since $\sigma$ is non-increasing, we have that

$$
\sigma(w) \leq \sigma(z) .
$$

Since $\sigma(r) /\left(1-r^{2}\right)^{1+\delta}$ is non-decreasing for some $\delta>0$, on the other hand, we have that

$$
\left(1-|z|^{2}\right)^{1+\delta} \sigma(w) \geq\left(1-|w|^{2}\right)^{1+\delta} \sigma(z) .
$$

Combining this with the relation $1-|z|^{2} \approx 1-|w|^{2}$ for $w \in E(z, r)$, we obtain that

$$
\left(1-|z|^{2}\right)^{1+\delta} \sigma(w) \gtrsim\left(1-|z|^{2}\right)^{1+\delta} \sigma(z)
$$

and so $\sigma(w) \gtrsim \sigma(z)$. These imply that $\sigma(w) \approx \sigma(z)$ for $w \in E(z, r)$. By (2.6), we have that

$$
|f(z)| \lesssim\{\sigma(z) A(E(z, r))\}^{-\frac{1}{p}}\|f\|_{\sigma} \approx\left\{\sigma(z)\left(1-|z|^{2}\right)^{2}\right\}^{-\frac{1}{p}}\|f\|_{\sigma}
$$

and so we get the first estimate (i).

Furthermore, the subharmonicity of $\left|f^{\prime}\right|^{p}$ gives that

$$
\left|f^{\prime}(z)\right|^{p} \lesssim \frac{1}{\left(1-|z|^{2}\right)^{2}} \int_{E(z, r)}\left|f^{\prime}(w)\right|^{p} d A(w) .
$$

By noting that $\sigma(w) \approx \sigma(z)$ and $1-|z|^{2} \approx 1-|w|^{2}$ for $w \in E(z, r)$, we have that

$$
\left|f^{\prime}(z)\right|^{p} \lesssim \frac{1}{\sigma(z)\left(1-|z|^{2}\right)^{2+p}} \int_{E(z, r)}\left|f^{\prime}(w)\right|^{p}\left(1-|w|^{2}\right)^{p} \sigma(w) d A(w) .
$$

From [1, Theorem 3.1], we see that

$$
\|f\|_{\sigma}^{p} \approx|f(0)|^{p}+\int_{\mathbb{D}}\left|f^{\prime}(z)\right|^{p}\left(1-|z|^{2}\right)^{p} \sigma(z) d A(z) .
$$

By applying (2.8) to the last integral in (2.7), we obtain the second estimate (ii).

In the above proof, we needed an equivalent norm (2.8) for $\|f\|_{\sigma}$. We also have another formula for $\|f\|_{\sigma}$. Note that the following lemma holds for any weight function $\sigma$ without conditions $\left(W_{1}\right) \sim\left(W_{3}\right)$.

Lemma 2.5. Let $p>0$ and $\sigma$ be a weight function. Then it holds that

$$
\|f\|_{\sigma}^{p} \approx|f(0)|^{p}+\int_{\mathbb{D}}|f(z)|^{p-2}\left|f^{\prime}(z)\right|^{2}\left\{\int_{|z|}^{1}\left(\log \frac{r}{|z|}\right) \sigma(r) r d r\right\} d A(z),
$$

for $f \in H(\mathbb{D})$. 
Proof. Recall that if $g$ is in the Hardy space $H^{p}$, then $|g|^{p}$ has the least harmonic majorant and it is equal to the Poisson integral

$$
P\left[\left|g^{*}\right|^{p}\right](z)=\int_{0}^{2 \pi} P\left(z, e^{i \theta}\right)\left|g^{*}\left(e^{i \theta}\right)\right| \frac{d \theta}{2 \pi},
$$

where $P\left(z, e^{i \theta}\right)=\operatorname{Re} \frac{e^{i \theta}+z}{e^{i \theta}-z}$ is the Poisson kernel for $\mathbb{D}$ and $g^{*}$ is the radial limit of $g$. Combining this with the Riesz Decomposition theorem, we have that

$$
|g(z)|^{p}=\int_{0}^{2 \pi} P\left(z, e^{i \theta}\right)\left|g^{*}\left(e^{i \theta}\right)\right|^{p} \frac{d \theta}{2 \pi}-\int_{\mathbb{D}} \log \left|\frac{1-\bar{w} z}{w-z}\right| d \mu_{|g|^{p}}(w) .
$$

Here $d \mu_{|g|^{p}}$ denotes the Riesz measure of $|g|^{p}$. In particular, we put $z=0$, then we obtain that

$$
\|g\|_{H^{p}}^{p}=|g(0)|^{p}+\int_{\mathbb{D}} \log \frac{1}{|w|} d \mu_{|g|^{p}}(w) .
$$

Since it is known that the Riesz measure of $|g|^{p}$ (see [14, p.1035 (3.2)]) is given by

$$
d \mu_{|g|^{p}}(w)=p^{2}|g(w)|^{p-2}\left|g^{\prime}(w)\right|^{2} d A(w),
$$

we have that

$$
\|g\|_{H^{p}}^{p}=|g(0)|^{p}+p^{2} \int_{\mathbb{D}}|g(w)|^{p-2}\left|g^{\prime}(w)\right|^{2} \log \frac{1}{|w|} d A(w),
$$

for $g \in H^{p}$.

Now we take an $f \in H(\mathbb{D})$ and $r \in(0,1)$. Since the dilated function $f_{r}(z)=$ $f(r z)$ is analytic in $\mathbb{D}$ and continuous on the closure of $\mathbb{D},(2.9)$ gives that

$$
\begin{aligned}
\int_{0}^{2 \pi}\left|f\left(r e^{i \theta}\right)\right|^{p} \frac{d \theta}{2 \pi} & =|f(0)|^{p}+p^{2} \int_{\mathbb{D}}|f(r w)|^{p-2}\left|f^{\prime}(r w)\right|^{2} r^{2} \log \frac{1}{|w|} d A(w) \\
& =|f(0)|^{p}+p^{2} \int_{r \mathbb{D}}|f(z)|^{p-2}\left|f^{\prime}(z)\right|^{2} \log \frac{r}{|z|} d A(z) .
\end{aligned}
$$

Multiplying the above formula by $2 r \sigma(r)$, integrating with respect to $r$ from 0 to 1 and applying Fubini's theorem, we get

$$
\begin{aligned}
\|f\|_{\sigma}^{p}=2|f(0)|^{p} & \int_{0}^{1} r \sigma(r) d r \\
& +2 p^{2} \int_{\mathbb{D}}|f(z)|^{p-2}\left|f^{\prime}(z)\right|^{2}\left\{\int_{|z|}^{1}\left(\log \frac{r}{|z|}\right) \sigma(r) r d r\right\} d A(z),
\end{aligned}
$$

which completes the proof.

In order to formulate our results, we need to introduce another weight function. For each weight $\sigma$, we put

$$
\omega_{\sigma}(z)=\int_{|z|}^{1}(t-|z|) \sigma(t) d t \quad(z \in \mathbb{D}) .
$$

Then we see that $\omega_{\sigma}$ is non-increasing, convex and $\omega_{\sigma}(z) \rightarrow 0$ as $|z| \rightarrow 1$. Furthermore $\omega_{\sigma}$ has the following property. 
Lemma 2.6. If $\sigma$ is an admissible Békollé weight function, then it holds that

$$
\omega_{\sigma}(r) \approx\left(1-r^{2}\right)^{2} \sigma(r)
$$

for every $r \in[0,1)$.

Proof. Since $\sigma$ is non-increasing, we have that

$$
\omega_{\sigma}(r)=\int_{r}^{1}(t-r) \sigma(t) d t \leq \frac{1}{2} \sigma(r)\left(1-r^{2}\right)^{2} .
$$

Since $\sigma(r) /\left(1-r^{2}\right)^{1+\delta}$ is non-decreasing for some $\delta>0$, we have that

$$
\begin{aligned}
\omega_{\sigma}(r) & \geq \frac{\sigma(r)}{\left(1-r^{2}\right)^{1+\delta}} \int_{r}^{1}(t-r)(1-t)^{1+\delta} d t \\
& \geq \frac{1}{2^{3+\delta}(2+\delta)(3+\delta)} \sigma(r)\left(1-r^{2}\right)^{2} .
\end{aligned}
$$

We accomplish the proof.

2.2. Composition operators on $A^{p}(\sigma d A)$. Now we show that each Möbius transformations $\varphi_{a}$ always induce a bounded composition operator on $A^{p}(\sigma d A)$. This property ensures that we may consider the operator $C_{\phi}$ under the assumption $\phi(0)=0$.

Proposition 2.7. Let $p>0, p_{0}>1$ and $\alpha>-1$. Suppose that $\sigma$ is an admissible Békollé weight function. For each $a \in \mathbb{D}, C_{\varphi_{a}}$ is a bounded composition operator on $A^{p}(\sigma d A)$.

Proof. Equation (2.8) and the change of variables formula give that

$$
\left\|C_{\varphi_{a}} f\right\|_{\sigma}^{p} \approx|f(a)|^{p}+\int_{\mathbb{D}}\left|f^{\prime}(z)\right|^{p}\left|\varphi_{a}^{\prime}\left(\varphi_{a}(z)\right)\right|^{p}\left(1-\left|\varphi_{a}(z)\right|^{2}\right)^{p} \sigma\left(\varphi_{a}(z)\right) J_{\varphi_{a}}(z) d A(z),
$$

where $J_{\varphi_{a}}(z)$ denotes the real Jacobian of $\varphi_{a}$ at $z$. By straightforward calculations we have that

$$
\left|\varphi_{a}^{\prime}\left(\varphi_{a}(z)\right)\right|^{p}\left(1-\left|\varphi_{a}(z)\right|^{2}\right)^{p-2} J_{\varphi_{a}}(z)=\left(1-|z|^{2}\right)^{p-2} .
$$

Lemma 2.6 shows that

$$
\left(1-\left|\varphi_{a}(z)\right|^{2}\right)^{2} \sigma\left(\varphi_{a}(z)\right) \approx \omega_{\sigma}\left(\varphi_{a}(z)\right) \text { and } \omega_{\sigma}(z) \approx\left(1-|z|^{2}\right)^{2} \sigma(z) .
$$

Since it follows from [8, Lemma 2.1] that

$$
\omega_{\sigma}(z) \approx \omega_{\sigma}\left(\varphi_{a}(z)\right) \quad(z \in \mathbb{D}),
$$

by an application of equation (2.8) once again, we obtain that

$$
\left\|C_{\varphi_{a}} f\right\|_{\sigma}^{p} \lesssim|f(a)|^{p}+\|f\|_{\sigma}^{p},
$$

for each $f \in A^{p}(\sigma d A)$. This implies that $C_{\varphi_{a}}\left(A^{p}(\sigma d A)\right) \subset A^{p}(\sigma d A)$. The closed graph theorem shows that $C_{\varphi_{a}}$ is bounded on $A^{p}(\sigma d A)$.

The following result is an immediate consequence of Proposition 2.7.

Corollary 2.8. Let $p>0, p_{0}>1$ and $\alpha>-1$. Suppose that $\sigma$ is an admissible Békollé weight function. Then any analytic self-map $\phi$ of $\mathbb{D}$ induces a bounded composition operator $C_{\phi}$ on $A^{p}(\sigma d A)$. 
The following change of variables formula help us in the arguments in our main results.

Lemma 2.9. Let $p>0, \phi$ be an analytic self-map of $\mathbb{D}$ and $\sigma$ a weight function. Then it holds that

$$
\|f \circ \phi\|_{\sigma}^{p} \approx|f(\phi(0))|^{p}+\int_{\mathbb{D}}|f(z)|^{p-2}\left|f^{\prime}(z)\right|^{2}\left\{\int_{0}^{1} N_{\phi}(r, z) \sigma(r) r d r\right\} d A(z),
$$

for $f \in H(\mathbb{D})$. Here $N_{\phi}(r, z)$ denotes the partial counting function for $\phi$ defined by

$$
N_{\phi}(r, z)=\sum_{w \in \phi^{-1}(z),|w| \leq r} \log \frac{r}{|w|} \quad(z \in \mathbb{D} \backslash\{\phi(0)\}, r \in(0,1)) .
$$

Proof. Recall Stanton's formula ([5, Theorem 2]) for integral means of subharmonic functions on $\mathbb{D}$. If $u$ is a positive subharmonic function on $\mathbb{D}$ and $\phi$ is an analytic self-map of $\mathbb{D}$, then it holds that

$$
\int_{0}^{2 \pi} u\left(\phi\left(r e^{i \theta}\right)\right) \frac{d \theta}{2 \pi}=u(\phi(0))+\int_{r \mathbb{D}} N_{\phi}(r, z) d \mu_{u}(z) \quad(r \in(0,1)),
$$

where $d \mu_{u}$ is the Riesz measure of $u$. Applying this formula to the positive subharmonic function $z \mapsto|f(z)|^{p}$, we obtain the desired formula.

Next we formulate the following sub-mean value property for the generalized counting function $\mathcal{N}_{\phi, \omega_{\sigma}}$. We will need this property in the proofs of our results.

Lemma 2.10. Let $t \in(0,1)$ be fixed and $\sigma$ a weight function. For any analytic self-map $\phi$ of $\mathbb{D}$ with $\phi(0)=0$, it holds that

$$
\mathcal{N}_{\phi, \omega_{\sigma}}(z) \lesssim \frac{1}{t^{2}\left(1-|z|^{2}\right)^{2}} \int_{E(z, t)} \mathcal{N}_{\phi, \omega_{\sigma}}(w) d A(w) \quad(t<|z|<1)
$$

Proof. Fix $z \in \mathbb{D} \backslash t \overline{\mathbb{D}}$ and $r \in(0,1)$. Since $N_{\phi}\left(r, \varphi_{z}(\cdot)\right)$ is subharmonic on $\mathbb{D} \backslash\left\{\varphi_{z}{ }^{-1}(0)\right\}=\mathbb{D} \backslash\{a\}$ and $t \overline{\mathbb{D}} \subset \mathbb{D} \backslash\{a\}$, we have that

$$
N_{\phi}(r, z)=N_{\phi}\left(r, \varphi_{z}(0)\right) \lesssim \frac{1}{t^{2}} \int_{t \overline{\mathbb{D}}} N_{\phi}\left(r, \varphi_{z}(w)\right) d A(w) .
$$

By the change of variables, we have that

$$
\int_{t \overline{\mathbb{D}}} N_{\phi}\left(r, \varphi_{z}(w)\right) d A(w)=\int_{E(z, t)} N_{\phi}(r, w) \frac{\left(1-|z|^{2}\right)^{2}}{|1-\bar{z} w|^{4}} d A(w) .
$$

Since $|1-\bar{z} w|^{4} \approx\left(1-|z|^{2}\right)^{4}$ for $w \in E(z, t)$, we obtain that

$$
N_{\phi}(r, z) \lesssim \frac{1}{t^{2}\left(1-|z|^{2}\right)^{2}} \int_{E(z, t)} N_{\phi}(r, w) d A(w)
$$

for $r \in(0,1)$. Multiplying the above inequality by $\sigma(r)$ and integrating with respect to $r$ from 0 to 1 , we get

$$
\int_{0}^{1} N_{\phi}(r, z) \sigma(r) d r \lesssim \frac{1}{t^{2}\left(1-|z|^{2}\right)^{2}} \int_{E(z, t)} d A(w) \int_{0}^{1} N_{\phi}(r, w) \sigma(r) d r .
$$


Now we fix $w \in E(z, t)$. Since $t<|z|<1$, we see that $0 \notin \overline{E(z, t)}$, and so $c:=\inf \{|v|: v \in \overline{E(z, t)}\}>0$ where $\overline{E(z, t)}=\{w \in \mathbb{D}:|\rho(w, z)| \leq t\}$. Since $\phi(0)=0$, Schwarz's lemma shows that each $u \in \mathbb{D}$ with $w=\phi(u)$ satisfies $c \leq|w| \leq|u|$. Thus we have the following inequalities

$$
\log \frac{r}{|u|} \leq \frac{1}{|u|}(r-|u|) \leq \frac{1}{c}(r-|u|)
$$

for $|u|<r<1$. These give that

$$
\int_{0}^{1} N_{\phi}(r, w) \sigma(r) d r=\sum_{w=\phi(u)} \int_{|u|}^{1} \log \frac{r}{|u|} \sigma(r) d r \leq \frac{1}{c} \mathcal{N}_{\phi, \omega_{\sigma}}(w) .
$$

On the other hand, the inequality $r-|u|<\log \frac{r}{|u|}$ for $|u|<r<1$ gives that

$$
\mathcal{N}_{\phi, \omega_{\sigma}}(z) \leq \int_{0}^{1} N_{\phi}(r, z) \sigma(r) d r .
$$

By inequalities $(2.11) \sim(2.13)$, we obtain the desired inequality.

At the end of this section, we quote the results on characterizations for the boundedness and compactness of $C_{\phi}: A^{p}\left(\sigma_{1} d A\right) \rightarrow A^{q}\left(\sigma_{2} d A\right)$ by O. Constantin.

Recently, Constantin [3, 4] obtained some properties of $A^{p}(\sigma d A)$ under condition $\left(W_{1}\right)$. In [4], Constantin proved the Carleson-type embedding theorem for $A^{p}(\sigma d A)$. For a given weight $\sigma$ and an analytic self-map $\phi$ of $\mathbb{D}$, we define a positive Borel measure $\mu$ by

$$
\mu(E)=\int_{\phi^{-1}(E)} \sigma(z) d A(z)
$$

for any Borel set $E$ of $\mathbb{D}$. Since it holds that

$$
\left\|C_{\phi} f\right\|_{\sigma}^{p}=\int_{\mathbb{D}}|f \circ \phi|^{p} \sigma d A=\int_{\mathbb{D}}|f|^{p} d \mu,
$$

Constantin's Carleson-type embedding theorem [4, Theorems 3.1 3.3] indicate the following results.

Theorem 2.11 ([4] Constantin). Let $p_{0}>1, \alpha>-1$ and $\sigma_{j}(j=1,2)$ be weight functions. Suppose that $\sigma_{1}(z) /\left(1-|z|^{2}\right)^{\alpha} \in B_{p_{0}}(\alpha)$. For $\lambda \in \mathbb{D}$ and $r \in(0,1)$, let $D_{\lambda, r}$ denote the disk $\{z \in \mathbb{D}:|z-\lambda|<r(1-|\lambda|)\}$. For any analytic self-map $\phi$ of $\mathbb{D}$, the followings hold.

(i) For $0<p \leq q<\infty, C_{\phi}: A^{p}\left(\sigma_{1} d A\right) \rightarrow A^{q}\left(\sigma_{2} d A\right)$ is bounded if and only if

$$
\int_{\phi^{-1}\left(D_{\lambda, r}\right)} \sigma_{2} d A=O\left(\left\{\int_{D_{\lambda, r}} \sigma_{1} d A\right\}^{q / p}\right) \quad(|\lambda| \rightarrow 1),
$$

and $C_{\phi}: A^{p}\left(\sigma_{1} d A\right) \rightarrow A^{q}\left(\sigma_{2} d A\right)$ is compact if and only if

$$
\int_{\phi^{-1}\left(D_{\lambda, r}\right)} \sigma_{2} d A=o\left(\left\{\int_{D_{\lambda, r}} \sigma_{1} d A\right\}^{q / p}\right) \quad(|\lambda| \rightarrow 1),
$$

for some $r \in(0,1)$. 
(ii) For $0<q<p<\infty, C_{\phi}: A^{p}\left(\sigma_{1} d A\right) \rightarrow A^{q}\left(\sigma_{2} d A\right)$ is bounded if and only if

$$
\mathbb{D} \ni \lambda \mapsto \frac{\int_{\phi^{-1}\left(D_{\lambda, r}\right)} \sigma_{2} d A}{\int_{D_{\lambda, r}} \sigma_{1} d A}
$$

belongs to $L^{p /(p-q)}\left(\sigma_{1} d A\right)$ for some $r \in(0,1)$. In this case $C_{\phi}$ is also compact.

Remark 2.12. In the above theorem, we see from the proof of it that the choice of $\{p, q\}$ is independent of $p_{0}$.

These results have the following corollary. It plays an important role in the proof of our results below, but its proof is very easy. Thus we state the result without the proof.

Corollary 2.13. Let $m$ be a positive integer. Under the same assumptions in Theorem 2.11, $C_{\phi}: A^{p}\left(\sigma_{1} d A\right) \rightarrow A^{q}\left(\sigma_{2} d A\right)$ is bounded (or compact) if and only if $C_{\phi}: A^{m p}\left(\sigma_{1} d A\right) \rightarrow A^{m q}\left(\sigma_{2} d A\right)$ is bounded (or compact, respectively).

\section{THE CASE $0<p \leq q<\infty$}

Theorem 3.1. Let $\sigma_{1}$ be an admissible Békollé weight function, $\sigma_{2}$ a weight function and $0<p \leq q<\infty$. For any analytic self-map $\phi$ of $\mathbb{D}, C_{\phi}: A^{p}\left(\sigma_{1} d A\right) \rightarrow$ $A^{q}\left(\sigma_{2} d A\right)$ is bounded if and only if

$$
\mathcal{N}_{\phi, \omega_{\sigma_{2}}}(z)=O\left(\omega_{\sigma_{1}}(z)^{\frac{q}{p}}\right) \quad(|z| \rightarrow 1) .
$$

Proof. First suppose that (3.1) holds and consider the case $q \geq 2$ and $\phi(0)=0$. By condition (3.1), we can choose a constant $K>0$ and $r_{0} \in[1 / 2,1)$ such that

$$
\mathcal{N}_{\phi, \omega_{\sigma_{2}}}(z) \leq K \omega_{\sigma_{1}}(z)^{\frac{q}{p}}, \quad z \in \mathbb{D} \backslash r_{0} \overline{\mathbb{D}} .
$$

For fixed $f \in A^{p}\left(\sigma_{1} d A\right)$, by Lemma 2.9, we have that

$$
\left\|C_{\phi} f\right\|_{\sigma_{2}}^{q} \lesssim|f(0)|^{q}+\int_{\mathbb{D}}|f(z)|^{q-2}\left|f^{\prime}(z)\right|^{2}\left\{\int_{0}^{1} N_{\phi}(r, z) \sigma_{2}(r) r d r\right\} d A(z) .
$$

Put

$$
\begin{aligned}
& I_{1}(f)=\int_{r_{0} \overline{\mathbb{D}}}|f(z)|^{q-2}\left|f^{\prime}(z)\right|^{2}\left\{\int_{0}^{1} N_{\phi}(r, z) \sigma_{2}(r) r d r\right\} d A(z), \\
& I_{2}(f)=\int_{\mathbb{D} \backslash r_{0} \overline{\mathbb{D}}}|f(z)|^{q-2}\left|f^{\prime}(z)\right|^{2}\left\{\int_{0}^{1} N_{\phi}(r, z) \sigma_{2}(r) r d r\right\} d A(z) .
\end{aligned}
$$

By noting our assumption $q-2 \geq 0$, Lemmas 2.4 (i) and 2.6 give that

$$
|f(z)|^{q-2} \lesssim \frac{\|f\|_{\sigma_{1}}^{q-2}}{\left\{\sigma_{1}(z)\left(1-|z|^{2}\right)^{2}\right\}^{\frac{q-2}{p}}} \approx \frac{\|f\|_{\sigma_{1}}^{q-2}}{\omega_{\sigma_{1}}(z)^{\frac{q-2}{p}}}
$$

By Lemmas 2.4 (ii) and 2.6, we have that

$$
\left|f^{\prime}(z)\right| \lesssim \frac{\|f\|_{\sigma_{1}}^{2}}{\left\{\sigma_{1}(z)\left(1-|z|^{2}\right)^{2}\right\}^{\frac{2}{p}}\left(1-|z|^{2}\right)^{2}} \approx \frac{\|f\|_{\sigma_{1}}^{2}}{\omega_{\sigma_{1}}(z)^{\frac{2}{p}}\left(1-|z|^{2}\right)^{2}}
$$


Hence it follows from (3.4) and (3.5) that

$$
I_{1}(f) \lesssim \max _{|z| \leq r_{0}} \frac{1}{\omega_{\sigma_{1}}(z)^{\frac{q}{p}}\left(1-|z|^{2}\right)^{2}}\|f\|_{\sigma_{1}}^{q} \int_{r_{0} \overline{\mathbb{D}}}\left\{\int_{0}^{1} N_{\phi}(r, z) \sigma_{2}(r) r d r\right\} d A(z) .
$$

Now we consider the function $g(z)=z+1$. Then $\left\|C_{\phi} g\right\|_{\sigma_{2}}^{q}=\|\phi+1\|_{\sigma_{2}}^{q} \leq 2^{q}$. By an application of Lemma 2.9 to $g$, we have that

$$
\int_{r_{0} \overline{\mathbb{D}}}\left\{\int_{0}^{1} N_{\phi}(r, z) \sigma_{2}(r) r d r\right\} d A(z) \lesssim 2^{q} .
$$

Combining this with (3.6), we have that

$$
I_{1}(f) \lesssim \max _{|z| \leq r_{0}} \frac{2^{q}}{\omega_{\sigma_{1}}(z)^{\frac{q}{p}}\left(1-|z|^{2}\right)^{2}}\|f\|_{\sigma_{1}}^{q} .
$$

Fix $z \in \mathbb{D} \backslash r_{0} \overline{\mathbb{D}}$. As in inequality (2.12), we obtain that

$$
\int_{0}^{1} N_{\phi}(r, z) \sigma_{2}(r) d r \leq \frac{1}{r_{0}} \mathcal{N}_{\phi, \omega_{\sigma_{2}}}(z)
$$

Combining this with (3.2), we have that

$$
I_{2}(f) \lesssim K \int_{\mathbb{D} \backslash r_{0} \overline{\mathbb{D}}}|f(z)|^{q-2}\left|f^{\prime}(z)\right|^{2} \omega_{\sigma_{1}}(z)^{\frac{q}{p}} d A(z) .
$$

By Lemma 2.4 and 2.6, we have that

$$
|f(z)|^{q-2} \lesssim \frac{\|f\|_{\sigma_{1}}^{q-p}}{\omega_{\sigma_{1}}(z)^{\frac{q-p}{p}}}|f(z)|^{p-2} .
$$

So we obtain that

$$
I_{2}(f) \lesssim K\|f\|_{\sigma_{1}}^{q-p} \int_{\mathbb{D}}|f(z)|^{p-2}\left|f^{\prime}(z)\right|^{2} \omega_{\sigma_{1}}(z) d A(z)
$$

Since it holds that

$$
\omega_{\sigma_{1}}(z)=\int_{|z|}^{1} \frac{1}{r}(r-|z|) \sigma_{1}(r) r d r \leq \int_{|z|}^{1}\left(\log \frac{r}{|z|}\right) \sigma_{1}(r) r d r,
$$

Lemma 2.5 gives that

$$
\int_{\mathbb{D}}|f(z)|^{p-2}\left|f^{\prime}(z)\right|^{2} \omega_{\sigma_{1}}(z) d A(z) \lesssim\|f\|_{\sigma_{1}}^{p},
$$

and so we obtain that $I_{2}(f) \lesssim K\|f\|_{\sigma_{1}}^{q}$. Combining this with (3.3) and (3.7), we see that $C_{\phi}\left(A^{p}\left(\sigma_{1} d A\right)\right) \subset A^{q}\left(\sigma_{2} d A\right)$, that is $C_{\phi}: A^{p}\left(\sigma_{1} d A\right) \rightarrow A^{q}\left(\sigma_{2} d A\right)$ is bounded by the closed graph theorem.

When the case $0<q<2$, we choose a positive integer $m$ such that $m q \geq 2$. Since the condition (3.1) implies that

$$
\mathcal{N}_{\phi, \omega_{\sigma_{2}}}(z)=O\left(\omega_{\sigma_{1}}(z)^{\frac{m q}{m p}}\right) \quad(|z| \rightarrow 1),
$$

the above arguments show that $C_{\phi}: A^{m p}\left(\sigma_{1} d A\right) \rightarrow A^{m q}\left(\sigma_{2} d A\right)$ is bounded. Thus it follows from Corollary 2.13 that $C_{\phi}: A^{p}\left(\sigma_{1} d A\right) \rightarrow A^{q}\left(\sigma_{2} d A\right)$ is also bounded. 
For the case $\phi(0) \neq 0$, we may consider the composition map $\Phi=\varphi_{\phi(0)} \circ \phi$. Then $\Phi(0)=0$. Equation (2.10) shows that

$$
\frac{\mathcal{N}_{\Phi, \omega_{\sigma_{2}}}(z)}{\omega_{\sigma_{1}}(z)^{q / p}}=\frac{\mathcal{N}_{\phi, \omega_{\sigma_{2}}}\left(\varphi_{\phi(0)}(z)\right)}{\omega_{\sigma_{1}}(z)^{q / p}} \approx \frac{\mathcal{N}_{\phi, \omega_{\sigma_{2}}}\left(\varphi_{\phi(0)}(z)\right)}{\omega_{\sigma_{1}}\left(\varphi_{\phi(0)}(z)\right)^{q / p}},
$$

for any $z \in \mathbb{D}$. Since $\left|\varphi_{\phi(0)}(z)\right| \rightarrow 1$ as $|z| \rightarrow 1$, we see that $\Phi$ also satisfies the condition (3.1), and so $C_{\Phi}: A^{p}\left(\sigma_{1} d A\right) \rightarrow A^{q}\left(\sigma_{2} d A\right)$ is bounded. By Proposition 2.7, $C_{\phi}=C_{\Phi} C_{\varphi_{\phi(0)}}$ is also bounded from $A^{p}\left(\sigma_{1} d A\right)$ into $A^{q}\left(\sigma_{2} d A\right)$.

Finally, we prove that (3.1) is a necessary condition for the boundedness of $C_{\phi}: A^{p}\left(\sigma_{1} d A\right) \rightarrow A^{q}\left(\sigma_{2} d A\right)$. Fix $z \in \mathbb{D}$ with $|z|>1 / 3$ and put

$$
f_{z}(w)=\frac{\left(1-|z|^{2}\right)^{\alpha+2-2 / p}}{\sigma_{1}(z)^{\frac{1}{p}}(1-\bar{z} w)^{\alpha+2}}, \quad w \in \mathbb{D} .
$$

By [4, Lemma 2.1] (see also [3, Lemma 3.1]), it holds that

$$
\int_{\mathbb{D}} \frac{1}{|1-\bar{z} w|^{p(\alpha+2)}} \sigma_{1}(w) d A(w) \approx \frac{\sigma_{1}(z)}{\left(1-|z|^{2}\right)^{p(\alpha+2)-2}},
$$

for $z \in \mathbb{D}$. So this implies that $f_{z} \in A^{p}\left(\sigma_{1} d A\right)$ and $\left\|f_{z}\right\|_{\sigma_{1}} \lesssim 1$. It follows from Lemma 2.9 that

$$
\begin{aligned}
\left\|C_{\phi} f_{z}\right\|_{\sigma_{2}}^{q} & \geq \int_{\mathbb{D}}\left|f_{z}(w)\right|^{q-2}\left|f_{z}^{\prime}(w)\right|^{2}\left\{\int_{0}^{1} N_{\phi}(r, w) \sigma_{2}(r) r d r\right\} d A(w) \\
& \geq \int_{\mathbb{D}}\left|f_{z}(w)\right|^{q-2}\left|f_{z}^{\prime}(w)\right|^{2} \mathcal{N}_{\phi, \omega_{\sigma_{2}}}(w) d A(w) \\
& \geq \int_{E\left(z, \frac{1-|z|}{2}\right)}\left|f_{z}(w)\right|^{q-2}\left|f_{z}^{\prime}(w)\right|^{2} \mathcal{N}_{\phi, \omega_{\sigma_{2}}}(w) d A(w) .
\end{aligned}
$$

Since $|1-\bar{z} w| \approx 1-|z|^{2}$ for $w \in E\left(z, \frac{1-|z|}{2}\right)$, Lemma 2.6 gives

$$
\left|f_{z}(w)\right|^{q-2}\left|f_{z}^{\prime}(w)\right|^{2} \approx \frac{(\alpha+2)^{2}|z|^{2}}{\omega_{\sigma_{1}}(z)^{\frac{q}{p}}\left(1-|z|^{2}\right)^{2}} .
$$

Combining this with (3.9), we have that

$$
\frac{1}{\omega_{\sigma_{1}}(z)^{\frac{q}{p}}} \frac{1}{\left(1-|z|^{2}\right)^{2}} \int_{E\left(z, \frac{1-|z|}{2}\right)} \mathcal{N}_{\phi, \omega_{\sigma_{2}}}(w) d A(w) \lesssim 1,
$$

for any $z \in \mathbb{D}$ with $|z|>1 / 3$. Since it holds that $|z|>\frac{1-|z|}{2}$ for $|z|>1 / 3$, Lemma 2.10 and (3.10), we obtain that

$$
\frac{\mathcal{N}_{\phi, \omega_{\sigma_{2}}}(z)}{\omega_{\sigma_{1}}(z)^{\frac{q}{p}}} \lesssim 1, \quad \text { for }|z|>1 / 3 .
$$

This implies that (3.1) is true. We accomplish the proof.

Next we will show that the compactness of $C_{\phi}: A^{p}\left(\sigma_{1} d A\right) \rightarrow A^{q}\left(\sigma_{2} d A\right)$ is characterized by the following condition

$$
N_{\phi, \omega_{\sigma_{2}}}(z)=o\left(\omega_{\sigma_{1}}(z)^{\frac{q}{p}}\right) \quad(|z| \rightarrow 1) .
$$


To prove this, we estimate the essential norm $\left\|C_{\phi}\right\|_{e}$ for the case $p \geq p_{0}, q \geq 2$ and $\phi(0)=0$. Since the essential norm $\left\|C_{\phi}\right\|_{e}$ is defined to be the distance from $C_{\phi}$ to the closed ideal of compact operators, that is

$$
\left\|C_{\phi}\right\|_{e}=\inf \left\{\left\|C_{\phi}-K\right\|: K \text { is compact from } A^{p}\left(\sigma_{1} d A\right) \text { into } A^{q}\left(\sigma_{2} d A\right)\right\},
$$

where $\left\|C_{\phi}-K\right\|$ denotes the operator norm of $C_{\phi}-K$, the compactness of $C_{\phi}$ is characterized by the condition $\left\|C_{\phi}\right\|_{e}=0$. Hence our object is to estimate $\left\|C_{\phi}\right\|_{e}$ in terms of $N_{\phi, \omega_{\sigma_{2}}}(z) / \omega_{\sigma_{1}}(z)^{q / p}$. For our aim, we need some preliminary results.

For the Taylor series expansion of $f \in H(\mathbb{D})$ and any integer $n \geq 1$ we put

$$
R_{n} f(z)=\sum_{k=n}^{\infty} a_{k} z^{k}, \quad z \in \mathbb{D},
$$

and $K_{n}=I-R_{n}$ where $I f=f$ is the identity operator. The following Lemma 3.2 and Corollary 3.3 hold for any weight function $\sigma$.

Lemma 3.2. If $1<p<\infty$ and $f \in A^{p}(\sigma d A)$, then $\left\|K_{n} f-f\right\|_{\sigma} \rightarrow 0$ as $n \rightarrow \infty$.

Proof. For $f \in A^{p}(\sigma d A)$ and $r \in(0,1)$, the dilated function $f_{r}$ is in the Hardy space $H^{p}$. Since $1<p<\infty$, Proposition 1 and Corollary 3 in [15] imply that there exists a constant $C>0$ such that

$$
\int_{0}^{2 \pi}\left|K_{n}\left(f_{r}\right)\left(e^{i \theta}\right)\right|^{p} \frac{d \theta}{2 \pi} \leq C \int_{0}^{2 \pi}\left|f_{r}\left(e^{i \theta}\right)\right|^{p} \frac{d \theta}{2 \pi},
$$

for all $r \in(0,1)$ and $n \geq 1$. Multiplying both sides by $2 r \sigma(r)$ and integrating with respect to $r$ from 0 to 1 give $\left\|K_{n} f\right\|_{\sigma}^{p} \leq C\|f\|_{\sigma}^{p}$, and so $\sup \left\{\left\|K_{n}\right\|: n \geq 1\right\}<\infty$. By applying [15, Proposition 1] once again, we have that $\left\|K_{n} f-f\right\|_{\sigma} \rightarrow 0$ as $n \rightarrow \infty$ for each $f \in A^{p}(\sigma d A)$.

Corollary 3.3. If $1<p<\infty$, then $\left\|R_{n} f\right\|_{\sigma} \rightarrow 0$ as $n \rightarrow \infty$ for each $f \in$ $A^{p}(\sigma d A)$. Moreover, $\sup \left\{\left\|R_{n}\right\|: n \geq 1\right\}<\infty$.

Proof. The second assertion of this corollary is verified by the principle of uniform boundedness. So we omit the details of the proof.

Lemma 3.4. Suppose that $1<p<\infty$ with $p \geq p_{0}, \alpha>-1$ and $\sigma$ is the weight function satisfied $\sigma(z) /\left(1-|z|^{2}\right)^{\alpha} \in B_{p_{0}}(\alpha)$. For each $f \in H(\mathbb{D})$, it holds that

$$
\left|R_{n} f(z)\right| \lesssim \frac{\|f\|_{\sigma}}{\left(\int_{\mathbb{D}} \sigma d A\right)^{1 / p}} \sum_{j=n}^{\infty} \frac{\Gamma(j+\alpha+2)}{j ! \Gamma(\alpha+2)}|z|^{j},
$$

for all $z \in \mathbb{D}$ and $n \geq 1$. Here $\Gamma(x)$ is the well-known Gamma function.

Proof. First note that the assumption $p \geq p_{0}$ implies that $\sigma(z) /\left(1-|z|^{2}\right)^{\alpha} \in B_{p}(\alpha)$ by Hölder's inequality. Since it follows from Theorem 2.1 that the Bergman projection $P_{\alpha}$ is bounded from $L^{p}(\sigma d A)$ onto $A^{p}(\sigma d A)$, we have that

$$
R_{n} f(z)=P_{\alpha}\left(R_{n} f\right)(z)=\int_{\mathbb{D}} \frac{R_{n} f(w)}{(1-\bar{w} z)^{\alpha+2}} d A_{\alpha}(w)
$$


Furthermore, by using the self-adjointness of $R_{n}$ and the expansion

$$
\frac{1}{(1-\bar{w} z)^{\alpha+2}}=\sum_{j=0}^{\infty} \frac{\Gamma(j+\alpha+2)}{j ! \Gamma(\alpha+2)}(\bar{w} z)^{j},
$$

we obtain that

$$
\left|R_{n} f(z)\right| \leq \sum_{j=n}^{\infty} \frac{\Gamma(j+\alpha+2)}{j ! \Gamma(\alpha+2)}|z|^{j} \int_{\mathbb{D}}|f(w)||w|^{j} d A_{\alpha}(w) .
$$

Hölder's inequality and the condition $\sigma(z) /\left(1-|z|^{2}\right)^{\alpha} \in B_{p}(\alpha)$ show that

$$
\begin{aligned}
\int_{\mathbb{D}}|f(w)||w|^{j} d A_{\alpha}(w) & \leq\left[\int_{\mathbb{D}}|f(w)|^{p} \sigma(w) d A(w)\right]^{\frac{1}{p}} \\
& \times\left[\int_{\mathbb{D}}|w|^{j p^{\prime}}\{\sigma(w)\}^{-\frac{p^{\prime}}{p}}\left(1-|z|^{2}\right)^{\alpha p^{\prime}} d A(w)\right]^{\frac{1}{p^{\prime}}} \\
& \lesssim \frac{\|f\|_{\sigma}}{\left(\int_{\mathbb{D}} \sigma d A\right)^{1 / p}}
\end{aligned}
$$

By (3.11) and (3.12) we obtain the desired estimation.

Theorem 3.5. Let $\sigma_{1}$ be an admissible Békollé weight function, $\sigma_{2}$ a weight function, $p_{0} \leq p \leq q<\infty$ and $q \geq 2$. Suppose that $\phi$ is an analytic self-map of $\mathbb{D}$ with $\phi(0)=0$ and $C_{\phi}: A^{p}\left(\sigma_{1} d A\right) \rightarrow A^{q}\left(\sigma_{2} d A\right)$ is bounded. Then it holds that

$$
\left\|C_{\phi}\right\|_{e}^{q} \approx \limsup _{|z| \rightarrow 1} \frac{\mathcal{N}_{\phi, \omega_{\sigma_{2}}}(z)}{\omega_{\sigma_{1}}(z)^{\frac{q}{p}}}
$$

Proof. First we prove the upper estimate. Since $C_{\phi}=C_{\phi} R_{n}+C_{\phi} K_{n}$ and $C_{\phi} K_{n}$ is compact, it holds that

$$
\left\|C_{\phi}\right\|_{e} \leq \liminf _{n \rightarrow \infty}\left\|C_{\phi} R_{n}\right\|
$$

Take $f \in A^{p}\left(\sigma_{1} d A\right)$ with $\|f\|_{\sigma_{1}} \leq 1$ and fix $t \in(1 / 2,1)$, arbitrarily. By Lemma 3.4 and the assumption $\phi(0)=0$ we have $\left|R_{n} f(\phi(0))\right|=0$, and so an application of Lemma 2.9 to $R_{n} f$ gives that

$$
\begin{aligned}
\left\|C_{\phi} R_{n} f\right\|_{\sigma_{2}}^{q} \lesssim & \int_{\{|z| \leq t\}}\left|R_{n} f(z)\right|^{q-2}\left|\left(R_{n} f\right)^{\prime}(z)\right|^{2}\left\{\int_{0}^{1} N_{\phi}(r, z) \sigma_{2}(r) r d r\right\} d A(z) \\
& +\int_{\{|z|>t\}}\left|R_{n} f(z)\right|^{q-2}\left|\left(R_{n} f\right)^{\prime}(z)\right|^{2}\left\{\int_{0}^{1} N_{\phi}(r, z) \sigma_{2}(r) r d r\right\} d A(z) .
\end{aligned}
$$

It follows from Lemmas 2.4, 2.6 and 3.4 that

$$
\begin{aligned}
& \left|R_{n} f(z)\right|^{q-2}\left|\left(R_{n} f\right)^{\prime}(z)\right|^{2} \\
& \lesssim \frac{\|f\|_{\sigma_{1}}^{q}}{\left(\int_{\mathbb{D}} \sigma_{1} d A\right)^{(q-2) / p}}\left(\sum_{j=n}^{\infty} \frac{\Gamma(j+\alpha+2)}{j ! \Gamma(\alpha+2)} t^{j}\right)^{q-2} \max _{|z| \leq t} \frac{1}{\omega_{\sigma_{1}}(z)^{2 / p}\left(1-|z|^{2}\right)^{2}}\left\|R_{n}\right\|^{2},
\end{aligned}
$$


for $|z| \leq t$. Since $\sup _{n \geq 1}\left\|R_{n}\right\|<\infty$ by Corollary 3.3 and

$$
\int_{\{|z| \leq t\}}\left\{\int_{0}^{1} N_{\phi}(r, z) \sigma_{2}(r) r d r\right\} d A(z)<\infty
$$

by the proof of Theorem 3.1, we see that the first integral in (3.15) converges to 0 as $n \rightarrow \infty$ uniformly on the unit ball of $A^{p}\left(\sigma_{1} d A\right)$.

On the other hand, the same argument as in the proof of Theorem 3.1 shows that

$$
\int_{0}^{1} N_{\phi}(r, z) \sigma_{2}(r) r d r \leq \frac{1}{t} \mathcal{N}_{\phi, \omega_{\sigma_{2}}}(z), \quad t \leq|z|<1
$$

and so we have that

$$
\begin{aligned}
& \int_{\{|z|>t\}}\left|R_{n} f(z)\right|^{q-2}\left|\left(R_{n} f\right)^{\prime}(z)\right|^{2}\left\{\int_{0}^{1} N_{\phi}(r, z) \sigma_{2}(r) r d r\right\} d A(z) \\
& \leq t \int_{\{|z|>t\}}\left|R_{n} f(z)\right|^{q-2}\left|\left(R_{n} f\right)^{\prime}(z)\right|^{2} \mathcal{N}_{\phi, \omega_{\sigma_{2}}}(z) d A(z) \\
& \leq t \sup _{|z|>t} \frac{\mathcal{N}_{\phi, \omega_{\sigma_{2}}}(z)}{\omega_{\sigma_{1}}(z)^{\frac{q}{p}}} \int_{\{|z|>t\}}\left|R_{n} f(z)\right|^{q-2}\left|\left(R_{n} f\right)^{\prime}(z)\right|^{2} \omega_{\sigma_{1}}(z)^{\frac{q}{p}} d A(z) .
\end{aligned}
$$

By Lemmas 2.4 and 2.6 we have that

$$
\left|R_{n} f(z)\right|^{q-2} \lesssim \frac{\left\|R_{n} f\right\|_{\sigma_{1}}^{q-p}}{\omega_{\sigma_{1}}(z)^{\frac{q-p}{p}}}\left|R_{n} f(z)\right|^{p-2} .
$$

Combining this with (3.16) and (3.8) and using Lemma 2.5, we obtain that

$$
\begin{aligned}
& \int_{\{|z|>t\}}\left|R_{n} f(z)\right|^{q-2}\left|\left(R_{n} f\right)^{\prime}(z)\right|^{2}\left\{\int_{0}^{1} N_{\phi}(r, z) \sigma_{2}(r) r d r\right\} d A(z) \\
& \lesssim \sup _{n \geq 1}\left\|R_{n}\right\|^{q} t \sup _{|z|>t} \frac{\mathcal{N}_{\phi, \omega_{\sigma_{2}}}(z)}{\omega_{\sigma_{1}}(z)^{\frac{q}{p}}},
\end{aligned}
$$

for any $n \geq 1$ and $t \in(1 / 2,1)$.

Taking the supremum over the unit ball of $A^{p}\left(\sigma_{1} d A\right)$ and letting $n \rightarrow \infty$ in (3.15), we have that

$$
\liminf _{n \rightarrow \infty}\left\|C_{\phi} R_{n}\right\|^{q} \lesssim t \sup _{|z|>t} \frac{\mathcal{N}_{\phi, \omega_{\sigma_{2}}}(z)}{\omega_{\sigma_{1}}(z)^{\frac{q}{p}}},
$$

for all $t \in(1 / 2,1)$. Letting $t \rightarrow 1$ in the above inequality, by (3.14), we obtain that

$$
\left\|C_{\phi}\right\|_{e}^{p} \lesssim \limsup _{|z| \rightarrow 1} \frac{\mathcal{N}_{\phi, \omega_{\sigma_{2}}}(z)}{\omega_{\sigma_{1}}(z)^{\frac{q}{p}}} .
$$

Next we prove the lower estimate in (3.13). For any sequence $\left\{z_{j}\right\} \subset \mathbb{D}$ with $\left|z_{j}\right| \rightarrow 1$ as $j \rightarrow \infty$, we put

$$
f_{z_{j}}(w)=\frac{\left(1-\left|z_{j}\right|^{2}\right)^{\alpha+2-2 / p}}{\sigma_{1}\left(z_{j}\right)^{\frac{1}{p}}\left(1-\overline{z_{j}} w\right)^{\alpha+2}}, \quad w \in \mathbb{D} .
$$


Then we see that $\left\{f_{z_{j}}\right\}$ is bounded in $A^{p}\left(\sigma_{1} d A\right)$ as in the proof of Theorem 3.1, and converges to 0 uniformly on compact subsets on $\mathbb{D}$ as $j \rightarrow \infty$. Lemma 2.3 implies that $\left\{f_{z_{j}}\right\}$ converges to 0 weakly in $A^{p}\left(\sigma_{1} d A\right)$, and so we see that $\left\|\mathcal{K} f_{z_{j}}\right\|_{\sigma_{2}} \rightarrow 0$ as $j \rightarrow \infty$ for all compact operators $\mathcal{K}: A^{p}\left(\sigma_{1} d A\right) \rightarrow A^{q}\left(\sigma_{2} d A\right)$. These facts give that

$$
\left\|C_{\phi}\right\|_{e}^{q} \gtrsim \lim _{j \rightarrow \infty}\left\|C_{\phi} f_{z_{j}}\right\|_{\sigma_{2}}^{q} .
$$

As in the proof of (3.9), Lemma 2.9 gives that

$$
\left\|C_{\phi} f_{z_{j}}\right\|_{\sigma_{2}}^{q} \gtrsim \int_{E\left(z_{j}, \frac{1-\left|z_{j}\right|}{2}\right)}\left|f_{z_{j}}(w)\right|^{q-2}\left|f_{z_{j}}^{\prime}(w)\right|^{2} \mathcal{N}_{\phi, \omega_{\sigma_{2}}}(w) d A(w),
$$

for all $j \geq 1$. By Lemma 2.6, it follows that

$$
\left|f_{z_{j}}(w)\right|^{q-2}\left|f_{z_{j}}^{\prime}(w)\right|^{2} \approx \frac{(\alpha+2)^{2}\left|z_{j}\right|^{2}}{\omega_{\sigma_{1}}\left(z_{j}\right)^{\frac{q}{p}}\left(1-\left|z_{j}\right|^{2}\right)^{2}},
$$

for $j \geq 1$ and $w \in E\left(z_{j}, \frac{1-\left|z_{j}\right|}{2}\right)$. Since $\left|z_{j}\right|>\frac{1-\left|z_{j}\right|}{2}$ if $j$ is sufficiently large, Lemma 2.10 gives that

$$
\mathcal{N}_{\phi, \omega_{\sigma_{2}}}\left(z_{j}\right) \lesssim \frac{1}{\left(1-\left|z_{j}\right|^{2}\right)^{2}} \int_{E\left(z_{j}, \frac{1-\left|z_{j}\right|}{2}\right)} \mathcal{N}_{\phi, \omega_{\sigma_{2}}}(w) d A(w) .
$$

By (3.18), (3.19) and (3.20) we obtain that

$$
\left|z_{j}\right|^{2} \frac{\mathcal{N}_{\phi, \omega_{\sigma_{2}}}\left(z_{j}\right)}{\omega_{\sigma_{1}}\left(z_{j}\right)^{\frac{q}{p}}} \lesssim\left\|C_{\phi} f_{z_{j}}\right\|_{\sigma_{2}}^{q}
$$

for sufficiently large $j$. Combining this with (3.17), we have that

$$
\lim _{j \rightarrow \infty} \frac{\mathcal{N}_{\phi, \omega_{\sigma_{2}}}\left(z_{j}\right)}{\omega_{\sigma_{1}}\left(z_{j}\right)^{\frac{q}{p}}} \lesssim\left\|C_{\phi}\right\|_{e}^{q}
$$

Since $\left\{z_{j}\right\} \subset \mathbb{D}$ with $\left|z_{j}\right| \rightarrow 1$ is arbitrarily, we get the desired lower estimate of $\left\|C_{\phi}\right\|_{e}$. This completes the proof.

Corollary 3.6. Let $\sigma_{1}$ be an admissible Békollé weight function, $\sigma_{2}$ a weight function and $0<p \leq q<\infty$. Suppose that $\phi$ is an analytic self-map of $\mathbb{D}$ which $C_{\phi}: A^{p}\left(\sigma_{1} d A\right) \rightarrow A^{q}\left(\sigma_{2} d A\right)$ is bounded. Then $C_{\phi}: A^{p}\left(\sigma_{1} d A\right) \rightarrow A^{q}\left(\sigma_{2} d A\right)$ is compact if and only if

$$
\mathcal{N}_{\phi, \omega_{\sigma_{2}}}(z)=o\left(\omega_{\sigma_{1}}(z)^{\frac{q}{p}}\right) \quad(|z| \rightarrow 1) .
$$

Proof. In the view of the proof of Theorem 3.1, we can assume that $\phi(0)=0$ without loss of generality. Since the case $p \geq p_{0}$ and $q \geq 2$ is an immediate consequence of Theorem 3.5, it is enough to prove that the case $0<p<p_{0}$ and $0<q<2$ because the rest of cases are verified by quite the same argument. Since we can choose a positive integer $m=m(p, q)$ such that $m p \geq p_{0}$ and $m q \geq 2$, we see that $C_{\phi}: A^{m p}\left(\sigma_{1} d A\right) \rightarrow A^{m q}\left(\sigma_{2} d A\right)$ is compact if and only if

$$
\mathcal{N}_{\phi, \omega_{\sigma_{2}}}(z)=o\left(\omega_{\sigma_{1}}(z)^{\frac{m q}{m p}}\right) \quad(|z| \rightarrow 1) .
$$


So, by an application of Corollary 2.13, we see that condition (3.21) also characterizes the compactness of $C_{\phi}: A^{p}\left(\sigma_{1} d A\right) \rightarrow A^{q}\left(\sigma_{2} d A\right)$ for the case $p<p_{0}$ and $q<2$.

\section{The CASE $0<q<p<\infty$}

In the proof of Theorem 4.1 below, we shall need Khinchine's inequality and the Hardy-Littlewood maximal function. Recall that the Rademacher functions $\left\{r_{j}(t)\right\}$ are defined by

$$
\begin{aligned}
& r_{0}(t)=\left\{\begin{array}{rc}
1 & 0 \leq t-[t]<\frac{1}{2}, \\
-1 & \frac{1}{2} \leq t-[t]<1,
\end{array}\right. \\
& r_{j}(t)=r_{0}\left(2^{j} t\right) \quad(j \geq 1) .
\end{aligned}
$$

Khinchine's Inequality. Let $0<p<\infty$. There are constants $0<A_{p} \leq B_{p}<$ $\infty$ such that, for any positive integer $m$ and any complex numbers $\left\{c_{j}\right\}_{j=1}^{m}$, it holds that

$$
A_{p}\left(\sum_{j=1}^{m}\left|c_{j}\right|^{2}\right)^{\frac{p}{2}} \leq \int_{0}^{1}\left|\sum_{j=1}^{m} c_{j} r_{j}(t)\right|^{p} d t \leq B_{p}\left(\sum_{j=1}^{m}\left|c_{j}\right|^{2}\right)^{\frac{p}{2}} .
$$

The Hardy-Littlewood Maximal Function. Let $\mathcal{M}[f]$ denote the HardyLittlewood maximal function for $f$, that is

$$
\mathcal{M}[f](z)=\sup _{\delta>0} \frac{1}{A(B(z, \delta))} \int_{B(z, \delta)}|f| d A,
$$

where $B(z, \delta)=\{w \in \mathbb{D}:|w-z|<\delta\}$. Since we can find a positive constant $c$ such that $E\left(z, \frac{1}{2}\right) \subset B\left(z, c\left(1-|z|^{2}\right)\right)$ for $z \in \mathbb{D}$, it holds that

$$
\frac{1}{\left(1-|z|^{2}\right)^{2}} \int_{E\left(z, \frac{1}{2}\right)}|f| d A \lesssim \mathcal{M}[f](z) \quad(z \in \mathbb{D}) .
$$

Moreover the Hardy-Littlewood maximal theorem (see [6, Theorem 4.3]) says that $\mathcal{M}[f] \in L^{p}$ and $\|\mathcal{M}[f]\|_{L^{p}} \lesssim\|f\|_{L^{p}}$ for $f \in L^{p}(1<p<\infty)$.

In our proof of Theorem 4.1, we adapt Luecking's approach in [10] or the method by Smith and Yang in [13] to weighted Bergman space with admissible Békollé weight. By using the same modification of Luecking's method and a $c$-adic decomposition of the disk $\mathbb{D}$, Constantin [4] proved the Carleson-type embedding theorem for $A^{p}(\sigma d A)$. In order to construct a suitable test function, however, we will use an $\varepsilon$-separated sequence of $\mathbb{D}$ instead of a $c$-adic decomposition of $\mathbb{D}$.

Theorem 4.1. Let $\sigma_{1}$ be an admissible Békollé weight function, $\sigma_{2}$ a weight function and $0<q<p<\infty$. For any analytic self-map $\phi$ of $\mathbb{D}, C_{\phi}: A^{p}\left(\sigma_{1} d A\right) \rightarrow$ $A^{q}\left(\sigma_{2} d A\right)$ is bounded if and only if

$$
\frac{\mathcal{N}_{\phi, \omega_{\sigma_{2}}}}{\omega_{\sigma_{1}}} \in L^{\frac{p}{p-q}}\left(\sigma_{1} d A\right) .
$$

Moreover, if $C_{\phi}: A^{p}\left(\sigma_{1} d A\right) \rightarrow A^{q}\left(\sigma_{2} d A\right)$ is bounded, then it is also compact in this case. 
Proof. By the same argument in proofs of Theorem 3.1 or Corollary 3.6, we may only prove the case $\phi(0)=0, p \geq p_{0}$ and $q \geq 2$.

First suppose that condition (4.2) holds and prove that $C_{\phi}: A^{p}\left(\sigma_{1} d A\right) \rightarrow$ $A^{q}\left(\sigma_{2} d A\right)$ is bounded. By Lemma 2.5 , it is enough to prove that

$$
\int_{\mathbb{D}}|f(z)|^{q-2}\left|f^{\prime}(z)\right|^{2}\left\{\int_{0}^{1} N_{\phi}(r, z) \sigma_{2}(r) r d r\right\} d A(z)<\infty
$$

for any $f \in A^{p}\left(\sigma_{1} d A\right)$. To prove this, we will divide the integral over $\mathbb{D}$ into two integrals over $\frac{1}{4} \overline{\mathbb{D}}$ and $\mathbb{D} \backslash \frac{1}{4} \overline{\mathbb{D}}$. As in the argument on inequality (3.7) in the proof of Theorem 3.1, however, we see that

$$
\begin{aligned}
& \int_{\frac{1}{4} \overline{\mathbb{D}}}|f(z)|^{q-2}\left|f^{\prime}(z)\right|^{2}\left\{\int_{0}^{1} N_{\phi}(r, z) \sigma_{2}(r) r d r\right\} d A(z) \\
\lesssim & \max _{|z| \leq \frac{1}{4}} \frac{1}{\omega_{\sigma_{1}}(z)^{\frac{q}{p}}\left(1-|z|^{2}\right)^{2}}\|f\|_{\sigma_{1}}^{q} .
\end{aligned}
$$

Hence we may only consider the integral over $\mathbb{D} \backslash \frac{1}{4} \overline{\mathbb{D}}$. Since $\phi(0)=0$, it follows from inequality (2.12) that

$$
\int_{0}^{1} N_{\phi}(r, z) \sigma_{2}(r) r d r \lesssim \mathcal{N}_{\phi, \omega_{\sigma_{2}}}(z)
$$

for $z \in \mathbb{D} \backslash \frac{1}{4} \overline{\mathbb{D}}$. Combining this with Lemma 2.10, we have that

$$
\begin{aligned}
& \int_{\mathbb{D} \backslash \frac{1}{4} \overline{\mathbb{D}}}|f(z)|^{q-2}\left|f^{\prime}(z)\right|^{2}\left\{\int_{0}^{1} N_{\phi}(r, z) \sigma_{2}(r) r d r\right\} d A(z) \\
\lesssim & \int_{\mathbb{D}}|f(z)|^{q-2}\left|f^{\prime}(z)\right|^{2} \frac{1}{\left(1-|z|^{2}\right)^{2}}\left\{\int_{E\left(z, \frac{1}{4}\right)} \mathcal{N}_{\phi, \omega_{\sigma_{2}}}(w) d A(w)\right\} d A(z) .
\end{aligned}
$$

By noting that $\chi_{E\left(z, \frac{1}{4}\right)}(w)=\chi_{E\left(w, \frac{1}{4}\right)}(z)$ and $1-|z|^{2} \approx 1-|w|^{2}$ for $w \in E\left(z, \frac{1}{4}\right)$, and applying Fubini's theorem, we have that

$$
\begin{aligned}
& \int_{\mathbb{D}}|f(z)|^{q-2}\left|f^{\prime}(z)\right|^{2} \frac{1}{\left(1-|z|^{2}\right)^{2}}\left\{\int_{E\left(z, \frac{1}{4}\right)} \mathcal{N}_{\phi, \omega_{\sigma_{2}}}(w) d A(w)\right\} d A(z) \\
\approx & \int_{\mathbb{D}}\left\{\int_{E\left(w, \frac{1}{4}\right)}|f(z)|^{q-2}\left|f^{\prime}(z)\right|^{2} d A(z)\right\} \frac{\mathcal{N}_{\phi, \omega_{\sigma_{2}}}(w)}{\left(1-|w|^{2}\right)^{2}} d A(w) .
\end{aligned}
$$

Since [13, Lemma 2.4] gives that

$$
\int_{E\left(w, \frac{1}{4}\right)}|f|^{q-2}\left|f^{\prime}\right|^{2} d A \lesssim \frac{1}{\left(1-|w|^{2}\right)^{2}} \int_{E\left(w, \frac{1}{2}\right)}|f|^{q} d A
$$

we obtain that

$$
\begin{aligned}
& \int_{\mathbb{D} \backslash \frac{1}{4} \overline{\mathbb{D}}}|f(z)|^{q-2}\left|f^{\prime}(z)\right|^{2}\left\{\int_{0}^{1} N_{\phi}(r, z) \sigma_{2}(r) r d r\right\} d A(z) \\
\lesssim & \int_{\mathbb{D}}\left\{\int_{E\left(w, \frac{1}{2}\right)}|f(z)|^{q} d A(z)\right\} \frac{\mathcal{N}_{\phi, \omega_{\sigma_{2}}}(w)}{\left(1-|w|^{2}\right)^{4}} d A(w) .
\end{aligned}
$$


By applying Fubini's theorem to the last formula in (4.3) once again, we have that

$$
\int_{\mathbb{D} \backslash \frac{1}{4} \overline{\mathbb{D}}}|f(z)|^{q-2}\left|f^{\prime}(z)\right|^{2}\left\{\int_{0}^{1} N_{\phi}(r, z) \sigma_{2}(r) r d r\right\} d A(z) \lesssim \int_{\mathbb{D}}|f(z)|^{q} H(z) d A(z),
$$

where

$$
H(z)=\int_{E\left(z, \frac{1}{2}\right)} \frac{\mathcal{N}_{\phi, \omega_{\sigma_{2}}}(w)}{\left(1-|w|^{2}\right)^{4}} d A(w) .
$$

Furthermore Hölder's inequality gives that

$$
\begin{aligned}
& \int_{\mathbb{D}}|f(z)|^{q} H(z) d A(z) \\
\leq & {\left[\int_{\mathbb{D}}|f(z)|^{p} \sigma_{1}(z) d A(z)\right]^{\frac{q}{p}} \cdot\left[\int_{\mathbb{D}} H(z)^{\frac{p}{p-q}} \sigma_{1}(z)^{-\frac{q}{p-q}} d A(z)\right]^{\frac{p-q}{p}} . }
\end{aligned}
$$

Since $\sigma_{1}(z) \approx \sigma_{1}(w)$ and $1-|z|^{2} \approx 1-|w|^{2}$ for $w \in E\left(z, \frac{1}{2}\right)$, it follows from Lemma 2.6 and inequality (4.1) that

$$
\begin{aligned}
H(z) & \lesssim \frac{\sigma_{1}(z)}{\left(1-|z|^{2}\right)^{2}} \int_{E\left(z, \frac{1}{2}\right)} \frac{\mathcal{N}_{\phi, \omega_{\sigma_{2}}}(w)}{\sigma_{1}(w)\left(1-|w|^{2}\right)^{2}} d A(w) \\
& \lesssim \frac{\sigma_{1}(z)}{\left(1-|z|^{2}\right)^{2}} \int_{E\left(z, \frac{1}{2}\right)} \frac{\mathcal{N}_{\phi, \omega_{\sigma_{2}}}(w)}{\omega_{\sigma_{1}}(w)} d A(w) \\
& \lesssim \sigma_{1}(z) \mathcal{M}\left[\frac{\mathcal{N}_{\phi, \omega_{\sigma_{2}}}}{\omega_{\sigma_{1}}}\right](z) \quad(z \in \mathbb{D}) .
\end{aligned}
$$

Thus the Hardy-Littlewood maximal theorem shows that

$$
\begin{aligned}
{\left[\int_{\mathbb{D}} H(z)^{\frac{p}{p-q}} \sigma_{1}(z)^{-\frac{q}{p-q}} d A(z)\right]^{\frac{p-q}{p}} } & \lesssim\left\|\mathcal{M}\left[\frac{\mathcal{N}_{\phi, \omega_{\sigma_{2}}}}{\omega_{\sigma_{1}}}\right]\right\|_{L^{\frac{p}{p-q}}\left(\sigma_{1} d A\right)} \\
& \lesssim\left\|\frac{\mathcal{N}_{\phi, \omega_{\sigma_{2}}}}{\omega_{\sigma_{1}}}\right\|_{L^{\frac{p}{p-q}}\left(\sigma_{1} d A\right)} .
\end{aligned}
$$

Inequalities (4.3) (4.6) and condition (4.2) imply that

$$
\int_{\mathbb{D}}|f(z)|^{q-2}\left|f^{\prime}(z)\right|^{2}\left\{\int_{0}^{1} N_{\phi}(r, z) \sigma_{2}(r) r d r\right\} d A(z)<\infty,
$$

and so $C_{\phi} f \in A^{q}\left(\sigma_{2} d A\right)$ for any $f \in A^{p}\left(\sigma_{1} d A\right)$. This indicates the boundedness of $C_{\phi}: A^{p}\left(\sigma_{1} d A\right) \rightarrow A^{q}\left(\sigma_{2} d A\right)$.

Conversely, we will prove that the boundedness of $C_{\phi}: A^{p}\left(\sigma_{1} d A\right) \rightarrow A^{q}\left(\sigma_{2} d A\right)$ gives condition (4.2). To do this, we choose an $\varepsilon$-separated sequence $\left\{z_{j}\right\} \subset \mathbb{D}$, that is

$$
\inf \left\{\left|\rho\left(z_{j}, z_{k}\right)\right|: j \neq k\right\}=\varepsilon>0 .
$$


By this condition, we can assume $\inf _{j \geq 1}\left|z_{j}\right|>0$ without loss of generality. Now we put

$$
\begin{aligned}
& g_{j}(z)=\frac{\left(1-\left|z_{j}\right|^{2}\right)^{\alpha+2-\frac{2}{p}}}{\sigma_{1}\left(z_{j}\right)^{\frac{1}{p}}} \cdot \frac{1}{\left(1-\overline{z_{j}} z\right)^{\alpha+2}} \quad(z \in \mathbb{D}, j \geq 1), \\
& f_{t}(z)=\sum_{j=1}^{\infty} \frac{c_{j} r_{j}(t)}{z_{j}} g_{j}(z) \quad(z \in \mathbb{D}, t \in[0,1))
\end{aligned}
$$

for some $\left\{c_{j}\right\} \in l^{p}$ and the Rademacher functions $\left\{r_{j}(t)\right\}$. Then these functions $f_{t}$ are in $A^{p}\left(\sigma_{1} d A\right)$ and

$$
\sup _{t \in[0,1)}\left\|f_{t}\right\|_{\sigma_{1}} \lesssim\left(\sum_{j=1}^{\infty}\left|c_{j}\right|^{p}\right)^{\frac{1}{p}}
$$

This inequality is verified by Theorems 2.1 and 2.2. In fact, Theorem 2.2 shows that

$$
\left\|f_{t}\right\|_{\sigma_{1}} \approx \sup \left\{\left|\left\langle f_{t}, h\right\rangle_{\alpha}\right|: h \in A^{p^{\prime}}\left(\sigma_{1}^{-\frac{p^{\prime}}{p}} d A_{\alpha p^{\prime}}\right),\|h\| \leq 1\right\} .
$$

Here $\|\cdot\|$ denotes the norm of the space $A^{p^{\prime}}\left(\sigma_{1}{ }^{-\frac{p^{\prime}}{p}} d A_{\alpha p^{\prime}}\right)$. Since

$$
\frac{\sigma_{1}(z)}{\left(1-|z|^{2}\right)^{\alpha}} \in B_{p}(\alpha) \Longleftrightarrow \frac{\sigma_{1}^{-\frac{p^{\prime}}{p}}(z)\left(1-|z|^{2}\right)^{\alpha p^{\prime}}}{\left(1-|z|^{2}\right)^{\alpha}} \in B_{p^{\prime}}(\alpha),
$$

Theorem 2.1 shows that $P_{\alpha}: L^{p^{\prime}}\left(\sigma_{1}^{-\frac{p^{\prime}}{p}} d A_{\alpha p^{\prime}}\right) \rightarrow A^{p^{\prime}}\left(\sigma_{1}^{-\frac{p^{\prime}}{p}} d A_{\alpha p^{\prime}}\right)$ is bounded and $h(w)=P_{\alpha} h(w)$ for $w \in \mathbb{D}$. Hence we have that

$$
\left\langle f_{t}, h\right\rangle_{\alpha}=\sum_{j=1}^{\infty} \frac{c_{j} r_{j}(t)}{z_{j}} \cdot \frac{\left(1-\left|z_{j}\right|^{2}\right)^{\alpha+2-\frac{2}{p}}}{\sigma_{1}\left(z_{j}\right)^{\frac{1}{p}}} \cdot \overline{h\left(z_{j}\right)} .
$$

As in the proof of Lemma 2.4, it holds that

$$
\left[\int_{E\left(z_{j}, r\right)} \sigma_{1}(z)^{-\frac{p^{\prime}}{p}} d A_{\alpha p^{\prime}}(z)\right]^{\frac{1}{p^{\prime}}} \approx \frac{\left(1-\left|z_{j}\right|^{2}\right)^{\alpha+2-\frac{2}{p}}}{\sigma_{1}\left(z_{j}\right)^{\frac{1}{p}}}
$$

for each $r \in(0,1)$ and $j \geq 1$. By (4.8), (4.9) and Hölder's inequality, we have that

$$
\begin{aligned}
\left|\left\langle f_{t}, h\right\rangle_{\alpha}\right| & \lesssim \sum_{j=1}^{\infty}\left|c_{j}\right|\left|h\left(z_{j}\right)\right|\left[\int_{E\left(z_{j}, r\right)} \sigma_{1}(z)^{-\frac{p^{\prime}}{p}} d A_{\alpha p^{\prime}}(z)\right]^{\frac{1}{p^{\prime}}} \\
& \leq\left[\sum_{j=1}^{\infty}\left|c_{j}\right|^{p}\right]^{\frac{1}{p}} \cdot\left[\sum_{j=1}^{\infty}\left|h\left(z_{j}\right)\right|^{p^{\prime}} \int_{E\left(z_{j}, r\right)} \sigma_{1}(z)^{-\frac{p^{\prime}}{p}} d A_{\alpha p^{\prime}}(z)\right]^{\frac{1}{p^{\prime}}}
\end{aligned}
$$

Since $\left\{z_{j}\right\}$ is $\varepsilon$-separated, [9, Theorem (3.12)] gives that

$$
\left[\sum_{j=1}^{\infty}\left|h\left(z_{j}\right)\right|^{p^{\prime}} \int_{E\left(z_{j}, r\right)} \sigma_{1}(z)^{-\frac{p^{\prime}}{p}} d A_{\alpha p^{\prime}}(z)\right]^{\frac{1}{p^{\prime}}} \lesssim\|h\|,
$$


and so we obtain the desired inequality (4.7).

Since $C_{\phi}: A^{p}\left(\sigma_{1} d A\right) \rightarrow A^{q}\left(\sigma_{2} d A\right)$ is bounded, (4.7) shows that

$$
\int_{\mathbb{D}}\left|\sum_{j=1}^{\infty} \frac{c_{j} r_{j}(t)}{z_{j}}\left(g_{j} \circ \phi\right)\right|^{q} \sigma_{2} d A \leq\left\|C_{\phi}\right\|^{q}\left\|f_{t}\right\|_{\sigma_{1}}^{q} \lesssim\left(\sum_{j=1}^{\infty}\left|c_{j}\right|^{p}\right)^{\frac{q}{p}} .
$$

By integrating the above inequalities from 0 to 1 with respect to $t$, and applying Fubini's theorem and Khinchine's inequality, we get

$$
\int_{\mathbb{D}}\left(\sum_{j=1}^{\infty}\left|\frac{c_{j}}{z_{j}}\left(g_{j} \circ \phi\right)\right|^{2}\right)^{\frac{q}{2}} \sigma_{2} d A \lesssim \int_{\mathbb{D}}\left(\int_{0}^{1}\left|\sum_{j=1}^{\infty} \frac{c_{j} r_{j}(t)}{z_{j}}\left(g_{j} \circ \phi\right)\right|^{q} d t\right) \sigma_{2} d A .
$$

Our assumption $q \geq 2$ shows that

$$
\begin{aligned}
\sum_{j=1}^{\infty} \frac{\left|c_{j}\right|^{q}}{\left|z_{j}\right|^{q}} \int_{\mathbb{D}}\left|g_{j} \circ \phi\right|^{q} \sigma_{2} d A & =\int_{\mathbb{D}} \sum_{j=1}^{\infty}\left|\frac{c_{j}}{z_{j}}\left(g_{j} \circ \phi\right)\right|^{q} \sigma_{2} d A \\
& \leq \int_{\mathbb{D}}\left(\sum_{j=1}^{\infty}\left|\frac{c_{j}}{z_{j}}\left(g_{j} \circ \phi\right)\right|^{2}\right)^{\frac{q}{2}} \sigma_{2} d A
\end{aligned}
$$

Combining these inequalities with Lemma 2.9, we obtain that

$$
\sum_{j=1}^{\infty} \frac{\left|c_{j}\right|^{q}}{\left|z_{j}\right|^{q}} \int_{\mathbb{D}}\left|g_{j}\right|^{q-2}\left|g_{j}^{\prime}\right|^{2} \mathcal{N}_{\phi, \omega_{\sigma_{2}}} d A \lesssim\left(\sum_{j=1}^{\infty}\left|c_{j}\right|^{p}\right)^{\frac{q}{p}}
$$

It holds that

$$
\frac{\left|z_{j}\right|^{2}}{\sigma_{1}\left(z_{j}\right)^{\frac{q}{p}}\left(1-\left|z_{j}\right|^{2}\right)^{2+2 \frac{q}{p}}} \lesssim\left|g_{j}\right|^{q-2}\left|g_{j}^{\prime}\right|^{2}
$$

on $E\left(z_{j}, \frac{1}{2}\right)$, and so

$$
\sum_{j=1}^{\infty}\left|c_{j}\right|^{q} \frac{\int_{E\left(z_{j}, \frac{1}{2}\right)} \mathcal{N}_{\phi, \omega_{\sigma_{2}}}(z) d A(z)}{\sigma_{1}\left(z_{j}\right)^{\frac{q}{p}}\left(1-\left|z_{j}\right|^{2}\right)^{2+2 \frac{q}{p}}} \lesssim\left(\sum_{j=1}^{\infty}\left|c_{j}\right|^{p}\right)^{\frac{q}{p}}<\infty .
$$

This inequality implies that the sequence

$$
\left\{\frac{\int_{E\left(z_{j}, \frac{1}{2}\right)} \mathcal{N}_{\phi, \omega_{\sigma_{2}}}(z) d A(z)}{\sigma_{1}\left(z_{j}\right)^{\frac{q}{p}}\left(1-\left|z_{j}\right|^{2}\right)^{2+2 \frac{q}{p}}}\right\}_{j \geq 1}
$$

belongs to the dual of $l^{\frac{p}{q}}$. Hence we see that

$$
\sum_{j=1}^{\infty}\left[\frac{\int_{E\left(z_{j}, \frac{1}{2}\right)} \mathcal{N}_{\phi, \omega_{\sigma_{2}}}(z) d A(z)}{\sigma_{1}\left(z_{j}\right)^{\frac{q}{p}}\left(1-\left|z_{j}\right|^{2}\right)^{2+2 \frac{q}{p}}}\right]^{\frac{p}{p-q}}<\infty .
$$

To derive the integrability condition (4.2) from this, we choose an $\varepsilon$-separated sequence $\left\{z_{j}\right\}$ in $\mathbb{D}$ such that the disks $E\left(z_{j}, \frac{1}{4}\right)$ cover $\mathbb{D}$. By Lemma 2.10, we have that

$$
\mathcal{N}_{\phi, \omega_{\sigma_{2}}}(z) \lesssim \frac{1}{\left(1-|z|^{2}\right)^{2}} \int_{E\left(z, \frac{1}{4}\right)} \mathcal{N}_{\phi, \omega_{\sigma_{2}}}(w) d A(w)
$$


for $z \in \mathbb{D} \backslash \frac{1}{4} \overline{\mathbb{D}}$. Then

$$
\begin{aligned}
\int_{\mathbb{D} \backslash \frac{1}{4} \overline{\mathbb{D}}}\left(\frac{\mathcal{N}_{\phi, \omega_{\sigma_{2}}}}{\omega_{\sigma_{1}}}\right)^{\frac{p}{p-q}} \sigma_{1} d A & \lesssim \int_{\mathbb{D} \backslash \frac{1}{4} \overline{\mathbb{D}}}\left(\frac{\int_{E\left(z, \frac{1}{4}\right)} \mathcal{N}_{\phi, \omega_{\sigma_{2}}}(w) d A(w)}{\omega_{\sigma_{1}}(z)\left(1-|z|^{2}\right)^{2}}\right)^{\frac{p}{p-q}} \sigma_{1}(z) d A(z) \\
& \leq \sum_{j=1}^{\infty} \int_{E\left(z_{j}, \frac{1}{4}\right)}\left(\frac{\int_{E\left(z, \frac{1}{4}\right)} \mathcal{N}_{\phi, \omega_{\sigma_{2}}}(w) d A(w)}{\omega_{\sigma_{1}}(z)\left(1-|z|^{2}\right)^{2}}\right)^{\frac{p}{p-q}} \sigma_{1}(z) d A(z)
\end{aligned}
$$

Since $1-|z|^{2} \approx 1-\left|z_{j}\right|^{2}, \sigma_{1}(z) \approx \sigma_{1}\left(z_{j}\right)$, and so $\omega_{\sigma_{1}}(z) \approx \omega_{\sigma_{1}}\left(z_{j}\right)$ for $z \in E\left(z_{j}, \frac{1}{2}\right)$, we have that

$$
\begin{aligned}
\left(\frac{\int_{E\left(z, \frac{1}{4}\right)} \mathcal{N}_{\phi, \omega_{\sigma_{2}}}(w) d A(w)}{\omega_{\sigma_{1}}(z)\left(1-|z|^{2}\right)^{2}}\right)^{\frac{p}{p-q}} \sigma_{1}(z) & \approx\left(\frac{\int_{E\left(z, \frac{1}{4}\right)} \mathcal{N}_{\phi, \omega_{\sigma_{2}}}(w) d A(w)}{\omega_{\sigma_{1}}\left(z_{j}\right)\left(1-\left|z_{j}\right|^{2}\right)^{2}}\right)^{\frac{p}{p-q}} \sigma_{1}\left(z_{j}\right) \\
& \leq\left(\frac{\int_{E\left(z_{j}, \frac{1}{2}\right)} \mathcal{N}_{\phi, \omega_{\sigma_{2}}}(w) d A(w)}{\omega_{\sigma_{1}}\left(z_{j}\right)\left(1-\left|z_{j}\right|^{2}\right)^{2}}\right)^{\frac{p}{p-q}} \sigma_{1}\left(z_{j}\right)
\end{aligned}
$$

for $z \in E\left(z_{j}, \frac{1}{4}\right)$. By noting that $A\left(E\left(z_{j}, \frac{1}{4}\right)\right) \approx\left(1-\left|z_{j}\right|^{2}\right)^{2}$ and applying Lemma 2.6 , we obtain that

$$
\begin{aligned}
\int_{\mathbb{D} \backslash \frac{1}{4} \overline{\mathbb{D}}}\left(\frac{\mathcal{N}_{\phi, \omega_{\sigma_{2}}}}{\omega_{\sigma_{1}}}\right)^{\frac{p}{p-q}} \sigma_{1} d A & \lesssim \sum_{j=1}^{\infty}\left(\frac{\int_{E\left(z_{j}, \frac{1}{2}\right)} \mathcal{N}_{\phi, \omega_{\sigma_{2}}}(w) d A(w)}{\omega_{\sigma_{1}}\left(z_{j}\right)\left(1-\left|z_{j}\right|^{2}\right)^{2}}\right)^{\frac{p}{p-q}} \sigma_{1}\left(z_{j}\right)\left(1-\left|z_{j}\right|^{2}\right)^{2} \\
& \approx \sum_{j=1}^{\infty}\left(\frac{\int_{E\left(z_{j}, \frac{1}{2}\right)} \mathcal{N}_{\phi, \omega_{\sigma_{2}}}(w) d A(w)}{\sigma_{1}\left(z_{j}\right)^{\frac{q}{p}}\left(1-\left|z_{j}\right|^{2}\right)^{2+\frac{2 q}{p}}}\right)^{\frac{p}{p-q}} \\
& <\infty
\end{aligned}
$$

Since the integrability on $\frac{1}{4} \overline{\mathbb{D}}$ is clear by the inequality

$$
\mathcal{N}_{\phi, \omega_{\sigma_{2}}}(z) \lesssim \int_{0}^{1} N_{\phi}(z, r) r \sigma_{2}(r) d r \leq \log \frac{1}{|z|},
$$

we obtain that

$$
\frac{\mathcal{N}_{\phi, \omega_{\sigma_{2}}}}{\omega_{\sigma_{1}}} \in L^{\frac{p}{p-q}}\left(\sigma_{1} d A\right)
$$

Finally we show that $C_{\phi}: A^{p}\left(\sigma_{1} d A\right) \rightarrow A^{q}\left(\sigma_{2} d A\right)$ is also compact. Take a bounded sequence $\left\{f_{j}\right\}$ in $A^{p}\left(\sigma_{1} d A\right)$ which converges to 0 uniformly on compact subsets of $\mathbb{D}$. By Lemma 2.3, we see that $\left\{f_{j}\right\}$ also converges to 0 weakly in $A^{p}\left(\sigma_{1} d A\right)$, and so it is enough to show that $\left\|C_{\phi} f_{j}\right\|_{\sigma_{2}} \rightarrow 0$ as $j \rightarrow \infty$. By Lemma 2.9 , this is equivalent to

$$
\lim _{j \rightarrow \infty} \int_{\mathbb{D}}\left|f_{j}(z)\right|^{q-2}\left|f_{j}^{\prime}(z)\right|^{2}\left\{\int_{0}^{1} N_{\phi}(r, z) \sigma_{2}(r) r d r\right\} d A(z)=0 .
$$

Since it holds that

$$
\int_{r \overline{\mathbb{D}}}\left\{\int_{0}^{1} N_{\phi}(r, z) \sigma_{2}(r) r d r\right\} d A(z)<\infty
$$


for any $r \in(0,1)$, we get that

$$
\lim _{j \rightarrow \infty} \int_{r \overline{\mathbb{D}}}\left|f_{j}(z)\right|^{q-2}\left|f_{j}^{\prime}(z)\right|^{2}\left\{\int_{0}^{1} N_{\phi}(r, z) \sigma_{2}(r) r d r\right\} d A(z)=0
$$

for any $r \in(0,1)$. As in the arguments in $(4.3) \sim(4.6)$, we have that

$$
\begin{aligned}
& \int_{\mathbb{D} \backslash r \overline{\mathbb{D}}}\left|f_{j}(z)\right|^{q-2}\left|f_{j}^{\prime}(z)\right|^{2}\left\{\int_{0}^{1} N_{\phi}(r, z) \sigma_{2}(r) r d r\right\} d A(z) \\
\lesssim & \int_{\mathbb{D} \backslash r \overline{\mathbb{D}}}\left|f_{j}(z)\right|^{q} \int_{E(z, 2 r)} \frac{\mathcal{N}_{\phi, \omega_{\sigma_{2}}}(w)}{\left(1-|w|^{2}\right)^{4}} d A(w) d A(z) \\
\lesssim & \left\|f_{j}\right\|_{\sigma_{1}}^{q}\left[\int_{\mathbb{D} \backslash r \overline{\mathbb{D}}}\left(\frac{\mathcal{N}_{\phi, \omega_{\sigma_{2}}}(w)}{\omega_{\sigma_{1}}(w)}\right)^{\frac{p}{p-q}} \sigma_{1}(w) d A(w)\right]^{\frac{p-q}{p}}
\end{aligned}
$$

for $r \in(0,1)$ and $j \geq 1$. Since the boundedness of $C_{\phi}$ implies that the last integral above can be made arbitrarily small by choosing $r$ sufficiently close to 1 , we obtain (4.10), and so the proof is complete.

Acknowledgement. We would like to thank the referee for careful reading the first version of the paper and helpful suggestions for corrections. The work of first author is a part of the research project sponsored by (NBHM)/DAE, India (Grant No.48/4/2009/R\&D-II/426). The second author is partly supported by the Grants-in-Aid for Young Scientists (B, No.23740100), Japan Society for the Promotion of Science (JSPS).

\section{REFERENCES}

1. A. Aleman and O. Constantin, Spectra of integration operators on weighted Bergman spaces, J. Anal. Math., 109 (2009), 199-231.

2. D. Békollé, Inégalité à poids pour le projecteur de Bergman dans la boule unité de $C^{n}$, Studia Math., 71 (1981/82), 305-323.

3. O. Constantin, Discretizations of integral operators and atomic decompositions in vectorvalued weighted Bergman spaces, Integral Equations Operator Theory, 59 (2007), 523-554.

4. O. Constantin, Carleson embeddings and some classes of operators on weighted Bergman spaces, J. Math. Anal. Appl., 365 (2010), 668-682.

5. M. Essén, D.F. Shea and C.S. Stanton, A value-distribution criterion for the class $L \log L$, and some related questions, Ann. Inst. Fourier (Grenoble), 35 (1985), 127-150.

6. J.B. Garnett, Bounded Analytic Functions, Revised First Edition, Graduate Texts in Mathematics Vol. 236, Springer, 2007.

7. F. Pérez-González, J. Rättyä and D. Vukotić, On composition operators acting beween Hardy and weighted Bergman spaces, Expo. Math., 25 (2007), 309-323.

8. K. Kellay and P. Lefèvre, Compact composition operators on weighted Hilbert spaces of analytic functions, J. Math. Anal. Appl., 386 (2012), 718-727.

9. D.H. Luecking, Representation and duality in weighted spaces of analytic functions, Indiana Univ. Math. J., 34 (1985), 319-336.

10. D.H. Luecking, Embedding theorems for spaces of analytic functions via Khinchine's inequality, Michigan Math. J., 40 (1993), 333-358.

11. J.H. Shapiro, The essential norm of a composition operator, Ann. of Math., 125 (1987), $375-404$.

12. W. Smith, Composition operators between Bergman and Hardy spaces, Trans. Amer. Math. Soc., 348 (1996), 2331-2348. 
13. W. Smith and L. Yang, Composition operators that improve integrability on weighted Bergman spaces, Proc. Amer. Math. Soc., 126 (1998), 411-420.

14. M. Stoll, A characterization of Hardy-Orlicz spaces on planar domains, Proc. Amer. Math. Soc., 117 (1993), 1031-1038.

15. K. Zhu, Duality of Bloch spaces and norm convergence of Taylor series, Michigan Math. J., 38 (1991), 89-101.

1 School of Mathematics, Shri Mata Vaishno Devi University, Kakryal, Katra182320, J\&K, INDIA.

E-mail address: aksju_76@yahoo.com

${ }^{2}$ Faculty of Engineering, Ibaraki University, Hitachi 316-8511, Japan.

E-mail address: sei-ueki@mx.ibaraki.ac.jp 